RESEARCH PAPER RP1244

Part of Journal of Research of the National Bureau of Standards, Volume 23, September 1939

\title{
SECOND SPECTRUM OF CHLORINE AND ITS STRUCTURE
}

\author{
By C. C. Kiess and T. L. deBruin
}

\section{ABSTRACT}

New wave lengths of $\mathrm{Cl} I$, extending from $9483 \mathrm{~A}$, in the infrared, to $2100 \mathrm{~A}$ in the ultraviolet, have been derived at the National Bureau of Standards from observations employing Geissler-tube and electrodeless discharges as light sources. These, together with unpublished observations of the Schumann region made by others, have been used to extend the analysis of the term system of Clu. New terms have been added to the partially known quintet and triplet systems, and the singlet system has been established. All three systems are linked together with intersystem combinations. Series of ${ }^{5} \mathrm{~S}$ and ${ }^{3} \mathrm{~S}$ terms, with ${ }^{4} \mathrm{~S}$ of $\mathrm{Cl}$ III as their limit, and of ${ }^{3} \mathrm{D}$ terms, with ${ }^{2} \mathrm{D}$ of $\mathrm{Cl}$ IIr as their limit, are in excellent agreement in fixing the value of the deepest term at $192,000 \mathrm{~cm}^{-1}$, whence an ionization potential of 23.70 volts is derived for $\mathrm{Cl}^{+}$.

CONTENTS

I. Introduction

II. Experimental procedure.

III. Results _......... 445

1. Wave lengths and intensities............ 445

2. Term structure

3. Series and ionization potential _...

4. Comparison with similar spectra

\section{IN'TRODUCTION}

The description and analysis of the second spectrum of chlorine presented in this paper are an outgrowth of the investigation, made 10 years ago at the National Bureau of Standards, ${ }^{1}$ of the spectrum emitted by neutral chlorine atoms. The observations on which that earlier work was based yielded a list of additional wave lengths that were recognized as characteristic of the spectrum emitted by singly ionized chlorine atoms. At that time a beginning had already been made on the analysis of $\mathrm{Cl}$ II-the spectral regularities first detected by Paulson ${ }^{2}$ among the visible and ultraviolet lines had been extended and interpreted by Paschen; ${ }^{3}$ and the triplet groups first observed by Hopfield ${ }^{4}$ in the Schumann region had been subsequently verified and extended by Bowen. ${ }^{5}$ To the lines classified by Paschen a new group had been added by the Blochs. ${ }^{6}$ The multiplets that we worked out from our new wave-length data were found to link together with those already known from the work of the earlier investigators; therefore, we decided to carry on the analysis to the extent warranted by the obtainable observational data.

\footnotetext{
'C. C. Kiess and T. I. deBruin, BS J. Research 2, 1117 (1929) RP73.

E. Paulson, Astrophys. J. 10, 299 (1914).

F. Paschen, Ann. Physik 71, 559 (1923).

- J. J. Hopfield, Phys. Rev. 20, 282 (1925).

I. S. Bowen, Phys. Rev. 31, 34 (1928).

L. and E. Bloch, Ann. physique [10] 8, 402 (1927).
} 
As the analysis progressed with the aid of our own and published wave lengths, notably those of the Blochs, we realized that the spectrum had not been sufficiently well observed to permit the theoretical term structure of $\mathrm{Cl}$ II to be worked out fully. Accordingly, a new series of observations was planned to cover those regions of the spectrum that hitherto had been inadequately observed.

While this work was in progress, Murakawa began the publication of the results of his observations and analysis of $\mathrm{Cl}$ II. His findings have been presented in a series of six papers. ${ }^{7}$ As Murakawa's analysis was, for the greater part, in harmony with our own, we felt that any additional publication on the subject should represent an analysis that was approximately complete. This status was nearly reached in 1932, at which time a description of our results was presented to the American Optical Society at its annual meeting. ${ }^{8}$ To aid in approaching this goal, we have had at our disposal the unpublished lists of observations of the extreme ultraviolet made by Bowen, by Weinberg, ${ }^{9}$ and by Boyce.

\section{EXPERIMENTAL PROCEDURE}

Both Geissler tubes and the electrodeless discharge were used to obtain the spectra emitted by ionized $\mathrm{Cl}$ atoms. The Geissler tubes and their behavior under different conditions of excitation have already been described in our paper on the arc spectrum. Although they emitted the visible portions of the spectrum with considerable intensity, they proved to be relatively weak in the violet and shorter regions owing, in part, to the scaly deposit that formed within the capillary of the tube. Furthermore, many of the lines excited by the highly condensed discharge were broad and fuzzy, the fainter ones being particularly difficult to measure.

To obtain a source emitting sharp lines, and intense in the ultraviolet, we adopted the electrodeless discharge, which has been employed so effectively by L. and E. Bloch, and others, in studying the spectra of the halogens and other elements. The tube was a Pyrex cylinder $12 \mathrm{~cm}$ long and $6 \mathrm{~cm}$ in diameter. To one end of it a fused-quartz window was cemented and to the other end was sealed the connection for the vacuum line. The chlorine was supplied by the decomposition of specially purified and dried $\mathrm{NaCl}$, about a gram of which was introduced within the tube shortly before the observations began. During operation, the tube was continuously open to the vacuum line, which was maintained by a Hickman ${ }^{10}$ diffusion pump supported by a forepump. The exciting field was maintained by the discharge of five condensers rated at $0.002 \mu \mathrm{f} \mathrm{each}$, and connected in parallel through a coil surrounding the tube and in series with a 2-cm spark gap. The coil consisted of 11 turns of No. 8 bare copper wire, each turn being $8 \mathrm{~cm}$ in diameter. The condensers were charged from a 40,000-volt

\footnotetext{
7 K. Murakawa, Sci. Papers Inst. Phys. Chem. Research (Tokyo), 15, 41 (1930); 15, 105 (1938); 20, 285 (1933). Z. Physik 69, 507 (1931);96, 117 (1935); 109, 173 (1938).

8 C. C. Kiess and T. L. deBruin, J. Opt. Soc. Am. 23, 121 (1933)

F. Weinberg, University of California Master's Dissertation (1925). "

10 K. C. D. Hickman and C. R. Sanford, Rev. Sci. Instr. 1, 140 (1930).
} 
transformer, the primary of which was connected to mains supplying current of 15 to $20 \mathrm{amp}$ at $110 \mathrm{v}$.

The spectra were photographed with the grating and quartz-prism spectrographs of the National Bureau of Standards. These instruments have been adequately described in previous publications. ${ }^{11}$ The 21-foot concave gratings ruled with $7,500,15,000$, and 20,000 lines per inch were used in recording the spectra between $2200 \mathrm{~A}$ in the ultraviolet and $9483 \mathrm{~A}$ in the infrared. The quartz-prism spectrographs were used to record the ultraviolet portion of the spectrum between 2000 and $3500 \mathrm{~A}$. Exposure times ranging from a few minutes up to 4 hours were required for the electrodeless discharge; but for the Geissler tubes longer exposure times, up to 20 hours, were needed. All of the spectrograms were also exposed to the iron are to obtain the standard wave lengths ${ }^{12}$ needed in the reductions.

When the 21-foot concave grating, ruled by Wood ${ }^{13}$ with 30,000 lines per inch, became available, some additional observations were made in order to resolve certain lines known to be complex from the results of the analysis. The source used for this final set of observations was a condensed discharge through a Pyrex Geissler tube.

The observations from which we obtained our first results for $\mathrm{Cl}$ II were made prior to the advent of the new types of Eastman photographic plates. For these earlier observations we used ordinary plates sensitized by bathing in dye solutions in order to record the regions of longer wave length. The new types of plates ${ }^{14}$ used for the observations of the electrodeless discharge and subsequent Geissler tube observations gave us an improved description of the long-wave portion of the spectrum that has furnished the clues for tying together the singlets and quintets with the more extensive triplet system.

\section{RESULTS}

\section{WAVE LENGTHS AND INTENSITIES}

The wave lengths that are characteristic of the spectrum emitted by singly ionized chlorine atoms are listed in the first column of tables 1 and 2. All of those extending from 9483 to $2100 \mathrm{~A}$ were derived from measurements of the spectrograms obtained at the National Bureau of Standards. They represent the means of from 2 to 10 or more measurements of the grating and prism observations. A few lines, measured on only one plate, have been corrected by amounts necessary to reduce the plate to the mean of the others. The intensities assigned to the lines are the usual visual estimates and are not comparable between widely separated regions of the spectrum. The letter $b$ following an intensity indicates that the line is diffuse in the electrodeless discharge; $d$ indicates that the line is double.

11 W. F. Meggers and K. Burns, BS Sci. Pap. 18, 191 (1922) S441.

12 Trans. Int. Astron. Union 3, 77 (1928).

13 R. W. Wood, Nature 140, 723 (1937)

14 C. E. K. Mees, J. Opt. Soc. Am. 23, 229 (1933). 
TABLE 1.-Wave lengths in the second spectrum of chlorine

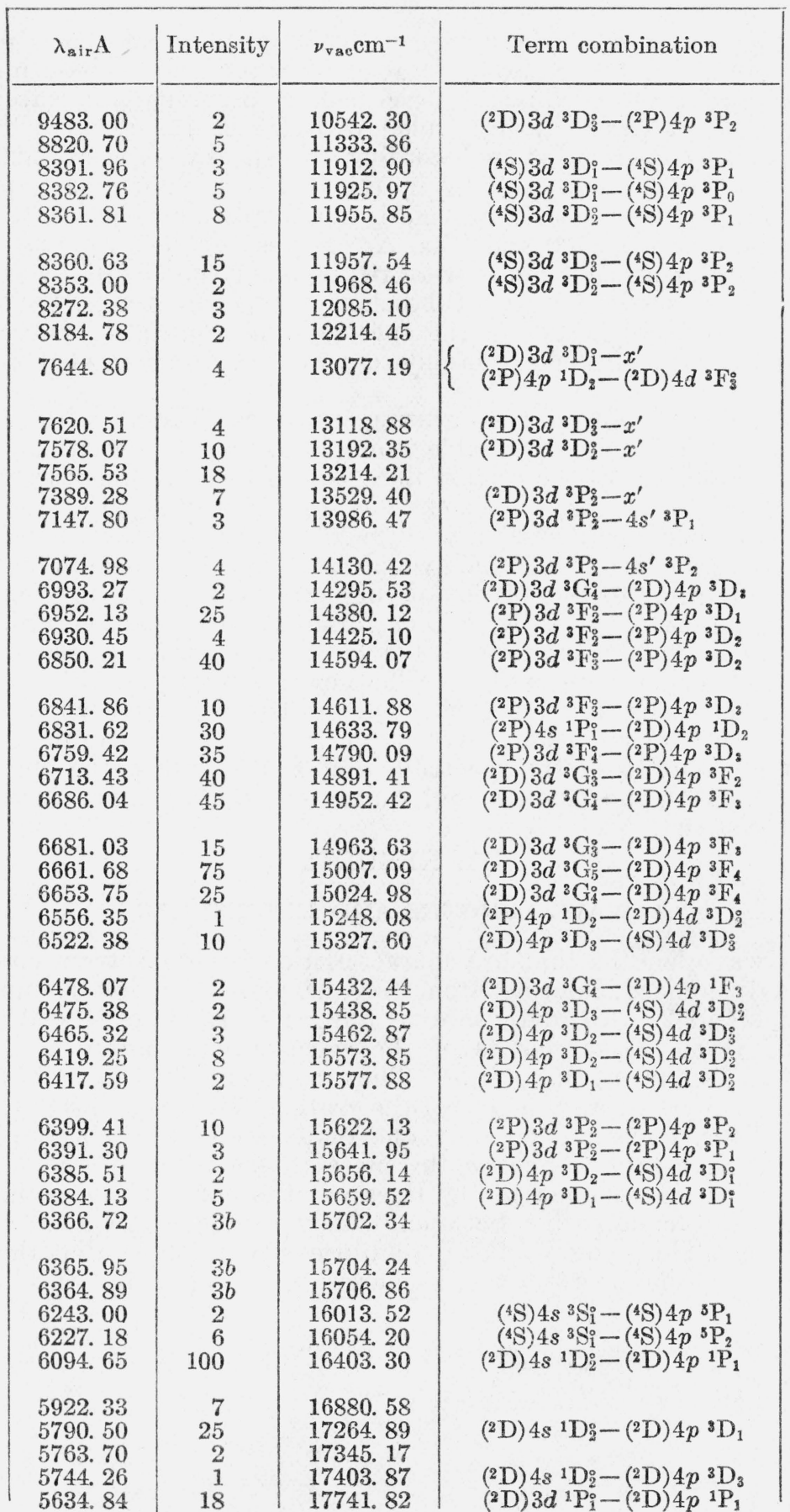


TABLE 1.-Wave lengths in the second spectrum of chlorine-Continued

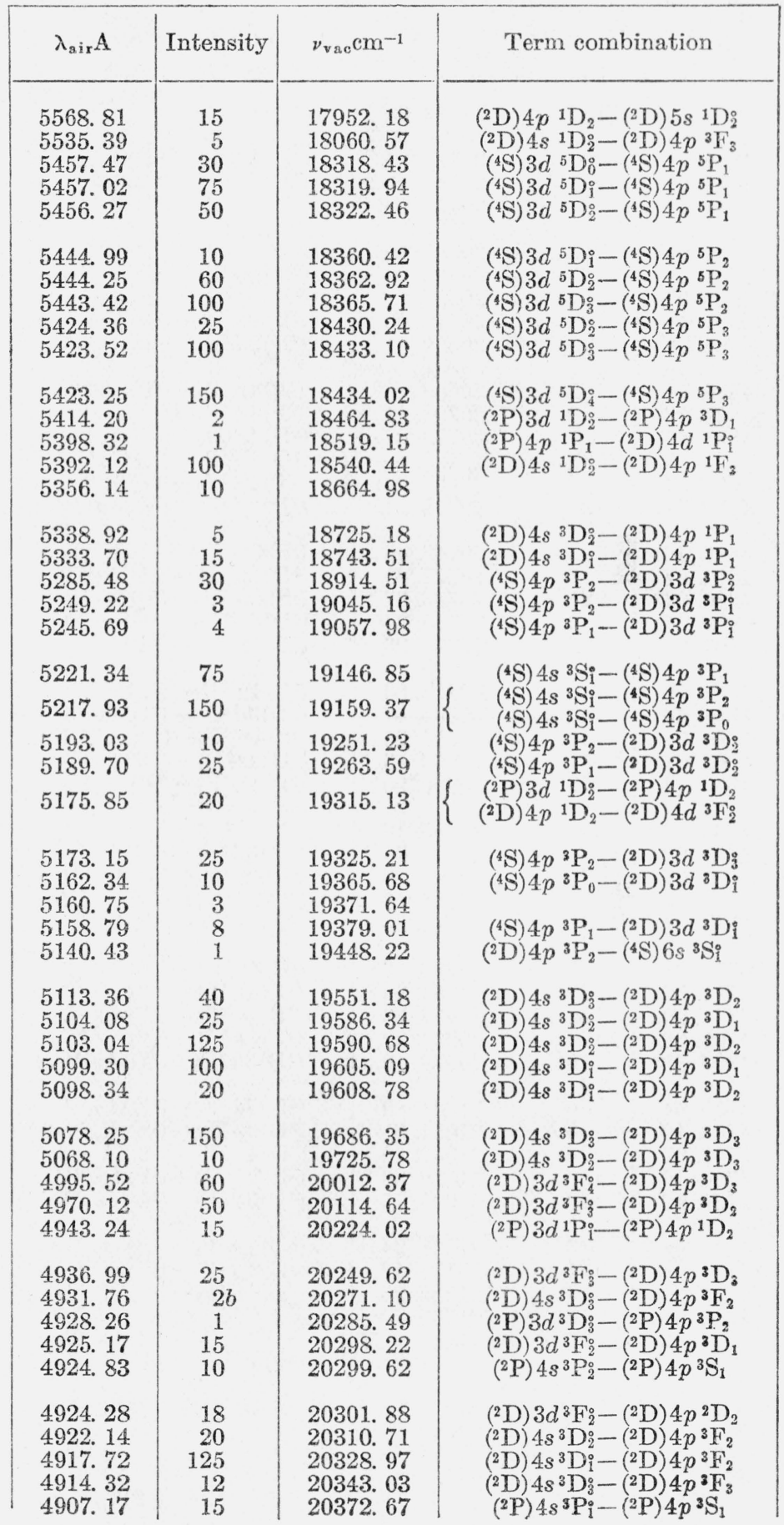


TABLE 1.-Wave lengths in the second spectrum of chlorine-Continued

\begin{tabular}{|c|c|c|c|}
\hline$\lambda_{\operatorname{sir}} \mathrm{A}$ & Intensity & $\nu_{\mathrm{vac}} \mathrm{cm}^{-1}$ & Term combination \\
\hline $\begin{array}{l}4904.76 \\
4898.94 \\
4896.77 \\
4891.62 \\
4877.70\end{array}$ & $\begin{array}{r}135 \\
7 \\
200 \\
4 \\
5\end{array}$ & $\begin{array}{l}\text { 20382. } 68 \\
20406.90 \\
20415.94 \\
20437.43 \\
20495.76\end{array}$ & $\begin{array}{l}\left.{ }^{2} \mathrm{D}\right) 4 s^{3} \mathrm{D}_{2}^{\circ}-\left({ }^{2} \mathrm{D}\right) 4 p^{3} \mathrm{~F}_{3} \\
\text { ('P) } 4 s^{3} \mathrm{P}_{0}^{\circ}-\left({ }^{2} \mathrm{P}\right) 4 p^{3} \mathrm{~S}_{1} \\
\text { (2D) } 4 s^{3} \mathrm{D}_{3}^{\circ}-\left({ }^{2} \mathrm{D}\right) 4 p^{3} \mathrm{~F}_{4} \\
\text { (2D) } 3 d^{3} \mathrm{~F}_{2}^{\circ}-\left({ }^{2} \mathrm{D}\right) 4 p^{3} \mathrm{D}_{3} \\
\text { (2D) } 4 p^{3} \mathrm{P}_{0}-\left({ }^{2} \mathrm{D}\right) 5 s^{3} \mathrm{D}_{\mathrm{I}}^{\circ}\end{array}$ \\
\hline $\begin{array}{l}4874.94 \\
4857.04 \\
4847.07 \\
4842.44 \\
4836.79\end{array}$ & $\begin{array}{r}2 \\
10 \\
4 \\
8 \\
20\end{array}$ & $\begin{array}{l}\text { 20507. } 36 \\
20582.94 \\
20625.28 \\
20645.00 \\
20669.11\end{array}$ & $\begin{array}{l}\text { (2D) } 4 p^{3} \mathrm{P}_{1}-\left({ }^{2} \mathrm{D}\right) 5 s^{3} \mathrm{D}_{2}^{\circ} \\
\left.{ }^{2} \mathrm{P}\right) 3 d^{3} \mathrm{D}_{2}^{\circ}-\left({ }^{2} \mathrm{P}\right) 4 p^{3} \mathrm{P}_{2} \\
\text { (2P) } 3 d^{3} \mathrm{D}_{2}^{\circ}-\left({ }^{2} \mathrm{P}\right) 4 p^{3} \mathrm{P}_{1} \\
\text { (2D) } 3 d^{3} \mathrm{~F}_{4}^{\circ}-\left({ }^{2} \mathrm{D}\right) 4 p^{3} \mathrm{~F}_{3}\end{array}$ \\
\hline $\begin{array}{l}4833.50 \\
4829.23 \\
4821.87\end{array}$ & $\begin{array}{l}2 \\
3 \\
2\end{array}$ & $\begin{array}{l}\text { 20683. } 18 \\
20701.47 \\
20733.07\end{array}$ & $\begin{array}{l}\left({ }^{2} \mathrm{P}\right) 4 p^{3} \mathrm{P}_{1}-\left({ }^{2} \mathrm{P}\right) 5 s^{3} \mathrm{P}_{0}^{0} \\
\text { (2P) } 4 p^{3} \mathrm{P}_{0}-\left({ }^{2} \mathrm{P}\right) 5 s^{3} \mathrm{P}_{1}^{0} \\
\text { (2D) } 4 s^{1} \mathrm{D}_{2}^{\circ}-\left({ }^{2} \mathrm{D}\right) 4 p^{3} \mathrm{P}_{2}\end{array}$ \\
\hline 4820.95 & 4 & 20737. 02 & $\begin{array}{l}\text { (2D) } 4 p^{3} \mathrm{P}_{2}-(2 \mathrm{D}) 5 s^{3} \mathrm{D}_{2} \\
\end{array}$ \\
\hline 4819. 79 & 25 & 20742.01 & (2D) $3 d^{3} \mathrm{~F}_{4}-\left({ }^{2} \mathrm{D}\right) 4 p^{3} \mathrm{~F}_{4}$ \\
\hline $\begin{array}{l}4819.46 \\
4811.57 \\
4810.06 \\
4809.05 \\
4807.68\end{array}$ & $\begin{array}{r}200 \\
12 \\
225 \\
9 \\
5\end{array}$ & $\begin{array}{l}20743.43 \\
20777.45 \\
20783.97 \\
20788.34 \\
20794.26\end{array}$ & 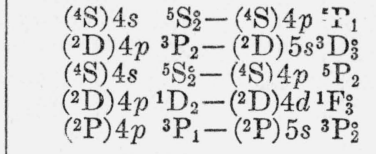 \\
\hline $\begin{array}{l}4803.16 \\
4798.40 \\
4794.54 \\
4792.04 \\
4785.44\end{array}$ & $\begin{array}{r}2 \\
15 \\
250 \\
12 \\
50\end{array}$ & $\begin{array}{l}20813.83 \\
20834.47 \\
20851.25 \\
20862.13 \\
20890.90\end{array}$ & 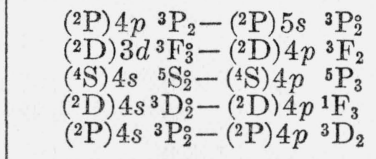 \\
\hline $\begin{array}{l}4784.46 \\
4781.82 \\
4781.32 \\
4780.02 \\
4778.93\end{array}$ & $\begin{array}{r}1 \\
50 \\
75 \\
1 \\
45\end{array}$ & $\begin{array}{l}\text { 20895. } 18 \\
\text { 20906. } 71 \\
20908.90 \\
\text { 20914. } 58 \\
20919.36\end{array}$ & $\begin{array}{l}\left({ }^{2} \mathrm{P}\right) 3 d^{3} \mathrm{D}_{1}^{\circ}-\left({ }^{2} \mathrm{P}\right) 4 p{ }^{3} \mathrm{P}_{2} \\
\left({ }^{2} \mathrm{D}\right) 3 d^{3} \mathrm{~F}_{3}^{3}-\left({ }^{2} \mathrm{D}\right) 4 p{ }^{3} \mathrm{~F}_{3} \\
\left({ }^{2} \mathrm{P}\right) 4 s^{3} \mathrm{P}_{2}^{0}-(2 \mathrm{P}) 4 p{ }^{3} \mathrm{D}_{3} \\
\left({ }^{2} \mathrm{P}\right) 3 d^{3} \mathrm{D}_{\mathrm{i}}-(2 \mathrm{P}) 4 p{ }^{3} \mathrm{P}_{1} \\
\left({ }^{2} \mathrm{P}\right) 4 s^{3} \mathrm{P}_{1}-\left({ }^{2} \mathrm{P}\right) 4 p{ }^{3} \mathrm{D}_{1}\end{array}$ \\
\hline $\begin{array}{l}4776.38 \\
4771.66 \\
4771.09 \\
4768.68 \\
4765.30\end{array}$ & $\begin{array}{r}5 \\
20 \\
40 \\
150 \\
10\end{array}$ & $\begin{array}{l}\text { 20930. } 52 \\
\text { 20951. } 23 \\
20953.73 \\
20964.32 \\
20979.19\end{array}$ & 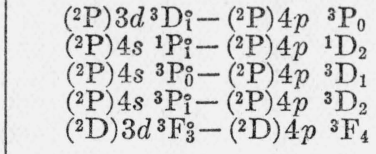 \\
\hline $\begin{array}{l}4755.64 \\
4753.49 \\
4748.67 \\
4740.40 \\
4739.42\end{array}$ & $\begin{array}{r}50 \\
8 \\
20 \\
150 \\
10\end{array}$ & $\begin{array}{l}21021.80 \\
21031.31 \\
21052.66 \\
21089.39 \\
21093.75\end{array}$ & 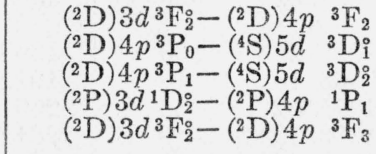 \\
\hline $\begin{array}{l}4738.41 \\
4721.43 \\
4714.28 \\
4713.51\end{array}$ & $\begin{array}{r}10 \\
25 \\
8 \\
3\end{array}$ & $\begin{array}{l}\text { 21098. } 24 \\
21174.12 \\
21206.23 \\
21209.70\end{array}$ & $\begin{array}{ll}\left.{ }^{2} \mathrm{D}\right) 4 p^{3} \mathrm{P}_{1}-\left({ }^{4} \mathrm{~S}\right) 5 d & { }^{3} \mathrm{D}_{1} \\
\left({ }^{2} \mathrm{D}\right) 4 p^{3} \mathrm{P}_{2}-\left({ }^{4} \mathrm{~S}\right) 5 d & { }^{3} \mathrm{D}_{3}^{3} \\
\left({ }^{2} \mathrm{D}\right) 4 p^{3} \mathrm{P}_{2}-\left({ }^{4} \mathrm{~S}\right) 5 d & { }^{3} \mathrm{D}_{2}^{\circ}\end{array}$ \\
\hline 4676.73 & 8 & 21376.50 & 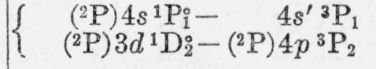 \\
\hline $\begin{array}{l}4656.16 \\
4627.68 \\
4624.36 \\
4610.59 \\
4607.72\end{array}$ & $\begin{array}{l}1 \\
2 \\
6 \\
1 \\
1\end{array}$ & $\begin{array}{l}21470.93 \\
21603.07 \\
21618.58 \\
21683.15 \\
21696.65\end{array}$ & $\left({ }^{4} \mathrm{~S}\right) 3 d^{5} \mathrm{D}_{3}^{\circ}-\left({ }^{4} \mathrm{~S}\right) 4 p^{3} \mathrm{P}_{2}$ \\
\hline
\end{tabular}


TABLE 1.-Wave lengths in the second spectrum of chlorine-Continued

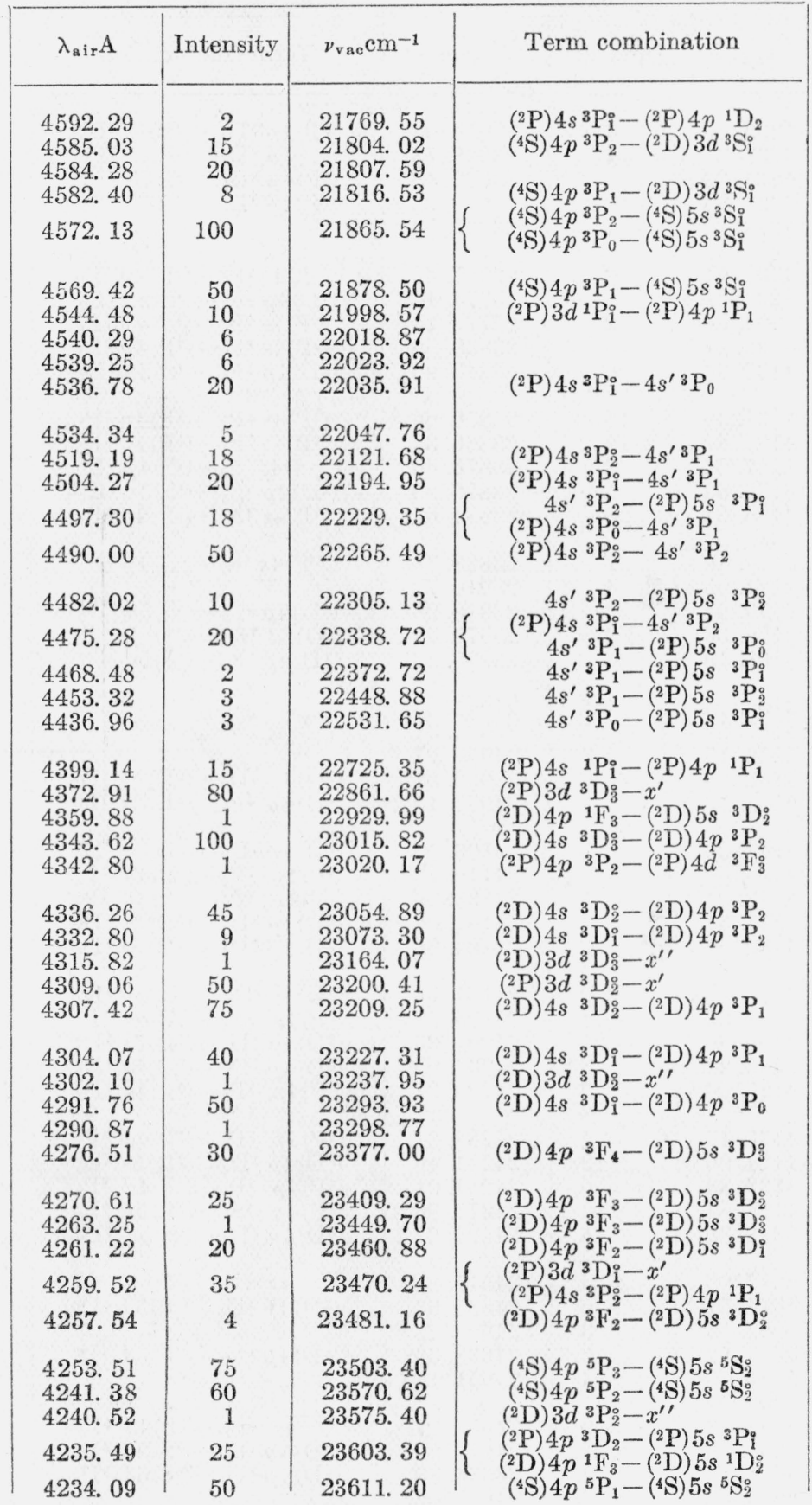


TABLE 1.-Wave lengths in the second spectrum of chlorine-Continued

\begin{tabular}{|c|c|c|c|}
\hline$\lambda_{\text {sir }} \mathrm{A}$ & Intensity & $\nu_{\mathrm{VAc}} \mathrm{cm}^{-1}$ & Term combination \\
\hline $\begin{array}{l}4233.60 \\
4227.37 \\
4224.92 \\
4221.80 \\
4218.76\end{array}$ & $\begin{array}{r}4 \\
4 \\
15 \\
3 \\
4\end{array}$ & $\begin{array}{l}23613.93 \\
23648.73 \\
23662.45 \\
23679.93 \\
23697.00\end{array}$ & 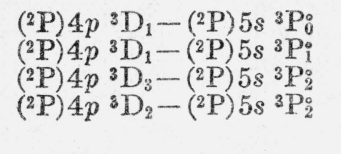 \\
\hline $\begin{array}{l}\text { 4208. } 03 \\
4205.07 \\
4204.54 \\
4195.11 \\
4.192 .24\end{array}$ & $\begin{array}{r}30 \\
10 \\
18 \\
18 \\
6\end{array}$ & $\begin{array}{l}23757.42 \\
23774.14 \\
23777.14 \\
23830.59 \\
23846.90\end{array}$ & $\begin{array}{l}\text { ('P) } 4 s^{3} \mathrm{P}_{2}-\left({ }^{2} \mathrm{P}\right) 4 p^{3} \mathrm{P}_{2} \\
\left({ }^{2} \mathrm{D}\right) 4 p^{3} \mathrm{~F}_{4}-\left({ }^{4} \mathrm{~S}\right) 5 d^{3} \mathrm{D}_{3} \\
\text { ('P) } 4 s^{3} \mathrm{P}_{2}-\left({ }^{2} \mathrm{P}\right) 4 p^{3} \mathrm{P}_{1} \\
\text { (2P) } 4 s^{3} \mathrm{P}_{1}-\left({ }^{2} \mathrm{P}\right) 4 p^{3} \mathrm{P}_{2} \\
\text { ('D) } 4 p^{3} \mathrm{~F}_{3}-\left({ }^{4} \mathrm{~S}\right) 5 d^{3} \mathrm{D}_{3}^{\circ}\end{array}$ \\
\hline $\begin{array}{l}4191.59 \\
4188.82 \\
4187.06 \\
4186.63 \\
4185.61\end{array}$ & $\begin{array}{r}15 \\
15 \\
2 \\
5 \\
20\end{array}$ & $\begin{array}{l}23850.60 \\
23866.37 \\
23876.40 \\
23878.85 \\
23884.67\end{array}$ & $\begin{array}{r}\left({ }^{2} \mathrm{P}\right) 4 s^{3} \mathrm{P}_{1}-\left({ }^{2} \mathrm{P}\right) 4 p^{3} \mathrm{P}_{1} \\
\left({ }^{2} \mathrm{P}\right) 4 s^{3} \mathrm{P}_{\mathrm{i}}-(2 \mathrm{P}) 4 p^{3} \mathrm{P}_{0} \\
\left({ }^{4} \mathrm{~S}\right) 4 s^{5} \mathrm{~S}_{2}^{\circ}-\left({ }^{4} \mathrm{~S}\right) 4 p^{3} \mathrm{P}_{1} \\
\left({ }^{2} \mathrm{D}\right) 4 p^{3} \mathrm{~F}_{3}-\left({ }^{4} \mathrm{~S}\right) 5 d^{3} \mathrm{D}_{2} \\
\left({ }^{2} \mathrm{P}\right) 4 s^{3} \mathrm{P}_{0}^{\circ}-\left({ }^{2} \mathrm{P}\right) 4 p^{3} \mathrm{P}_{1}\end{array}$ \\
\hline $\begin{array}{l}\text { 4184. } 89 \\
\text { 4181. } 05 \\
4179.61 \\
4170.66 \\
4166.10\end{array}$ & $\begin{array}{l}7 \\
4 \\
2 \\
8 \\
4\end{array}$ & $\begin{array}{l}23888.78 \\
23910.72 \\
23918.96 \\
23970.29 \\
23996.52\end{array}$ & $\begin{array}{l}\left({ }^{4} \mathrm{~S}\right) 4 s^{5} \mathrm{~S}_{2}-\left({ }^{4} \mathrm{~S}\right) 4 p^{3} \mathrm{P}_{2} \\
\left({ }^{2} \mathrm{D}\right) 4 p^{3} \mathrm{~F}_{2}-\left({ }^{4} \mathrm{~S}\right) 5 d^{3} \mathrm{D}_{3}^{3} \\
\left({ }^{2} \mathrm{D}\right) 3 d^{1} \mathrm{D}_{2}^{\circ}-\left({ }^{2} \mathrm{D}\right) 4 p{ }^{1} \mathrm{P}_{1} \\
\text { (2D) } 4 p{ }^{3} \mathrm{~F}_{2}-\left({ }^{4} \mathrm{~S}\right) 5 d^{3} \mathrm{D}_{\mathrm{i}}^{\circ}\end{array}$ \\
\hline $\begin{array}{l}\text { 4157. } 98 \\
\text { 4157. } 82 \\
4156.15 \\
4153.98 \\
4151.12\end{array}$ & $\begin{array}{r}5 \\
25 \\
7 \\
2 \\
1\end{array}$ & $\begin{array}{l}\text { 24043. } 39 \\
24044.31 \\
24053.97 \\
24066.54 \\
24083.12\end{array}$ & $\begin{array}{l}\left({ }^{2} \mathrm{D}\right) 4 p{ }^{3} \mathrm{D}_{3}-\left({ }^{2} \mathrm{D}\right) 5 s^{3} \mathrm{D}_{2} \\
\left({ }^{2} \mathrm{D}\right) 4 p{ }^{3} \mathrm{~F}_{3}-\left({ }^{2} \mathrm{D} 5\right) s{ }^{1} \mathrm{D}_{2}^{8}\end{array}$ \\
\hline $\begin{array}{l}\text { 4147. } 09 \\
4146.47 \\
4143.04 \\
4134.31 \\
4133.66\end{array}$ & $\begin{array}{r}30 \\
1 \\
5 \\
4 \\
20\end{array}$ & $\begin{array}{l}24106.52 \\
24110.12 \\
24130.09 \\
24181.04 \\
24184.84\end{array}$ & 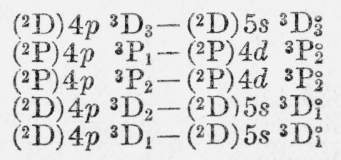 \\
\hline $\begin{array}{l}4132.48 \\
4131.80 \\
4130.86 \\
4130.22 \\
4125.96\end{array}$ & $\begin{array}{r}200 \\
1 \\
25 \\
8 \\
3\end{array}$ & $\begin{array}{l}24191.74 \\
24195.73 \\
24201.23 \\
24204.98 \\
24229.97\end{array}$ & 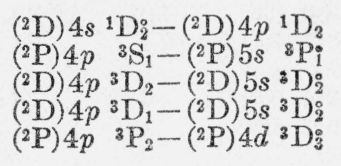 \\
\hline $\begin{array}{l}\text { 4125. } 17 \\
4124.00 \\
4122.38 \\
4118.84 \\
4079.88\end{array}$ & $\begin{array}{r}1 \\
12 \\
0 \\
4 \\
15\end{array}$ & $\begin{array}{l}24234.61 \\
24241.49 \\
24251.01 \\
24271.86 \\
24503.63\end{array}$ & 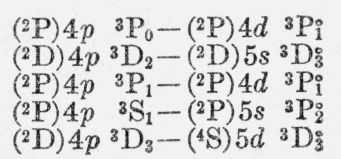 \\
\hline $\begin{array}{l}4077.93 \\
4074.51 \\
4062.53 \\
4057.52 \\
4055.46\end{array}$ & $\begin{array}{l}4 \\
6 \\
3 \\
6 \\
4\end{array}$ & $\begin{array}{l}24515.35 \\
24535.93 \\
24608.28 \\
24638.66 \\
24651.18\end{array}$ & $\begin{array}{r}4 s^{\prime}{ }^{3} \mathrm{P}_{2}-\left({ }^{2} \mathrm{P}\right) 4 d{ }^{3} \mathrm{~F}_{2} \\
\text { ('D) } 4 p^{3} \mathrm{D}_{3}-\left({ }^{4} \mathrm{~S}\right) 5 d^{3} \mathrm{D}_{2}^{\circ} \\
\left({ }^{2} \mathrm{D}\right) 4 p^{3} \mathrm{D}_{2}-\left({ }^{4} \mathrm{~S}\right) 5 d^{2} \mathrm{D}_{8}\end{array}$ \\
\hline $\begin{array}{l}4054.18 \\
4052.22 \\
4051.58 \\
4049.08 \\
4044.65\end{array}$ & $\begin{array}{r}9 \\
12 \\
4 \\
2 \\
4\end{array}$ & $\begin{array}{l}24658.97 \\
24670.89 \\
24674.78 \\
24690.02 \\
24717.06\end{array}$ & 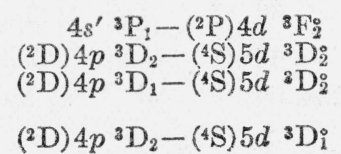 \\
\hline
\end{tabular}


TABLI 1.-Wave lengths in the second specirum of chlorine-Continued

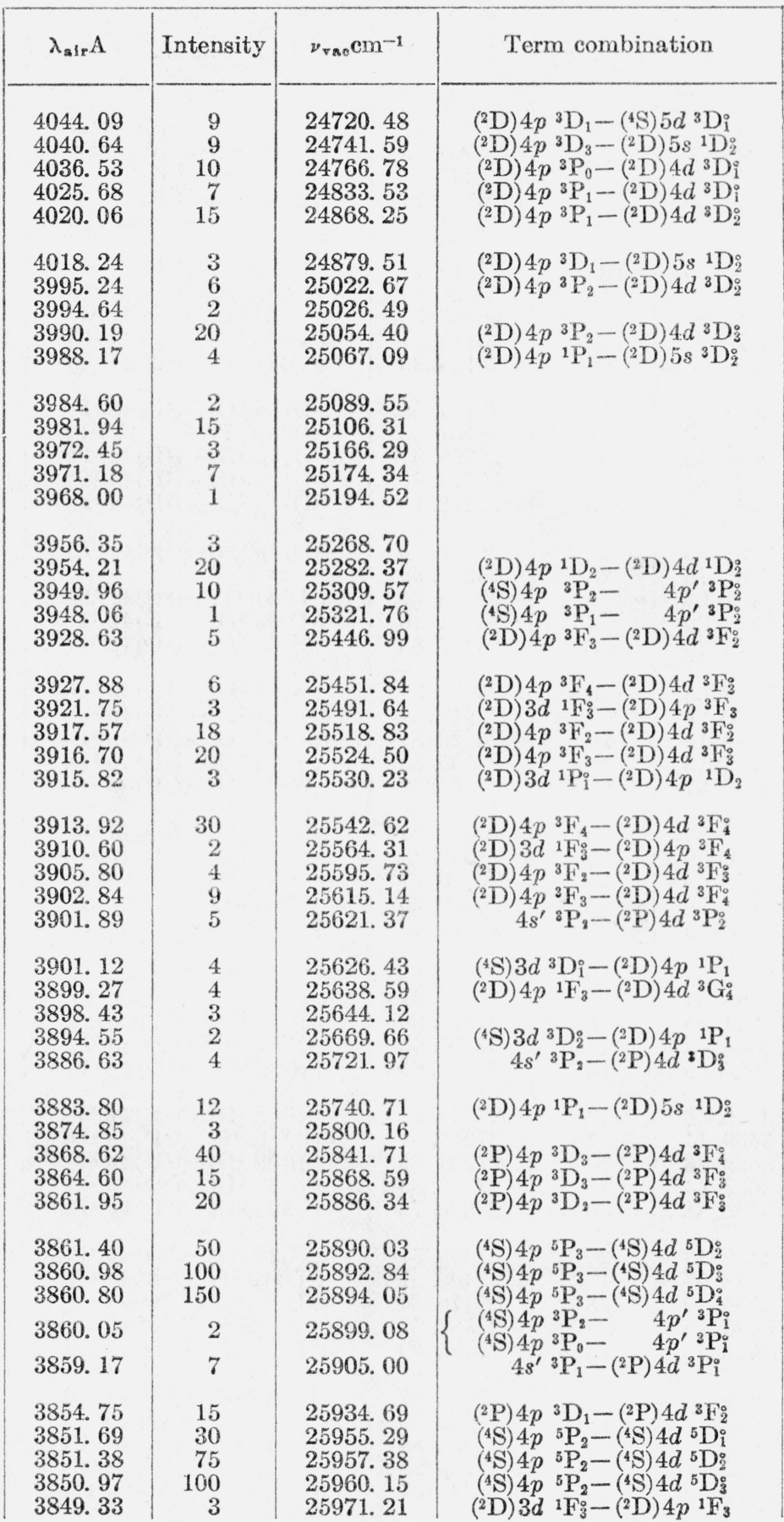


TABLE 1.-Wave lengths in the second spectrum of chlorine-Continued

\begin{tabular}{|c|c|c|c|}
\hline$\lambda_{\mathrm{Bir}} \mathrm{A}$ & Intensity & $\nu_{\mathrm{VBe}} \mathrm{cm}^{-1}$ & Term combination \\
\hline $\begin{array}{l}3845.84 \\
3845.69 \\
3845.42 \\
3843.26 \\
3838.37\end{array}$ & $\begin{array}{r}30 \\
75 \\
50 \\
100 \\
20\end{array}$ & $\begin{array}{l}25994.77 \\
25995.79 \\
25997.61 \\
26012.22 \\
26045.36\end{array}$ & $\begin{array}{l}\left({ }^{4} \mathrm{~S}\right) 4 p^{5}{ }^{5} \mathrm{P}_{1}-\left({ }^{4} \mathrm{~S}\right) 4 d^{5} \mathrm{D}_{0}^{\circ} \\
\left.{ }^{4} \mathrm{~S}\right) 4 p{ }^{5} \mathrm{P}_{1}-\left({ }^{4} \mathrm{~S}\right) 4 d d^{5} \mathrm{D}_{1}^{\circ} \\
\left({ }^{4} \mathrm{~S}\right) 4 p p^{5} \mathrm{P}_{1}-\left({ }^{4} \mathrm{~S}\right) 4 d^{5} \mathrm{D}_{2}^{\circ} \\
\left({ }^{2} \mathrm{P}\right) 3 d d^{1} \mathrm{P}_{1}^{\circ}-\left({ }^{2} \mathrm{P}\right) 4 p p^{1} \mathrm{~S}_{0} \\
\left({ }^{2} \mathrm{D}\right) 4 p^{3} \mathrm{~F}_{4}-\left({ }^{2} \mathrm{D}\right) 4 d^{3}{ }^{3} \mathrm{G}_{4}^{\circ}\end{array}$ \\
\hline $\begin{array}{l}3833.40 \\
3830.80 \\
3829.27 \\
3827.62 \\
3820.25\end{array}$ & $\begin{array}{r}200 \\
15 \\
15 \\
150 \\
100\end{array}$ & $\begin{array}{l}26079.13 \\
26096.83 \\
26107.26 \\
26118.51 \\
26168.90\end{array}$ & $\begin{array}{l}\text { (2D) } 4 p^{3} \mathrm{~F}_{4}-\left({ }^{2} \mathrm{D}\right) 4 d^{3} \mathrm{G}_{5} \\
\text { (2D) } 4 p^{3} \mathrm{~F}_{3}-(2 \mathrm{D}) 4 d^{3} \mathrm{G}_{3} \\
\text { ('D) } 3 d^{1} \mathrm{D}_{2}-\left({ }^{2} \mathrm{D}\right) 4 p^{1} \mathrm{~F}_{3} \\
\text { (2D) } 4 p^{3} \mathrm{~F}_{3}-(2 \mathrm{D}) 4 d^{3} \mathrm{G}_{4} \\
\text { (2D) } 4 p^{3} \mathrm{~F}_{2}-\left({ }^{2} \mathrm{D}\right) 4 d^{3}{ }^{3} \mathrm{G}_{3}\end{array}$ \\
\hline $\begin{array}{l}3818.40 \\
3815.43 \\
3810.10 \\
3809.51 \\
3805.24\end{array}$ & $\begin{array}{r}30 \\
1 \\
30 \\
40 \\
75\end{array}$ & $\begin{array}{l}26181.58 \\
26201.96 \\
26238.61 \\
26242.67 \\
26272.13\end{array}$ & 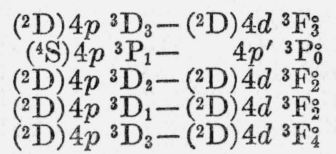 \\
\hline $\begin{array}{l}3798.80 \\
3793.75 \\
3781.23 \\
3776.20 \\
3774.25\end{array}$ & $\begin{array}{r}50 \\
25 \\
30 \\
4 \\
25\end{array}$ & $\begin{array}{l}26316.66 \\
26351.69 \\
26438.94 \\
26474.16 \\
26487.83\end{array}$ & $\begin{array}{l}\left({ }^{2} \mathrm{D}\right) 4 p{ }^{3} \mathrm{D}_{2}-\left({ }^{2} \mathrm{D}\right) 4 d^{3} \mathrm{~F}_{3} \\
\text { (2D) } 4 p{ }^{1} \mathrm{~F}_{3}-\left({ }^{2} \mathrm{D}\right) 4 d{ }^{1} \mathrm{~F}_{3} \\
\text { ('D } 4 s^{3} \mathrm{D}_{3}^{\circ}-\left({ }^{2} \mathrm{D}\right) 4 p{ }^{1} \mathrm{D}_{2} \\
\left({ }^{4} \mathrm{~S}\right) 3 d^{3} \mathrm{D}_{1}^{\circ}-\left({ }^{2} \mathrm{D}\right) 4 p{ }^{3} \mathrm{D}_{1}\end{array}$ \\
\hline $\begin{array}{l}3773.68 \\
3770.69 \\
3769.13 \\
3768.13 \\
3767.57\end{array}$ & $\begin{array}{r}20 \\
1 \\
20 \\
18 \\
30\end{array}$ & $\begin{array}{l}26491.84 \\
26512.84 \\
26523.81 \\
26530.85 \\
26534.80\end{array}$ & 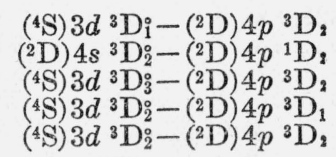 \\
\hline $\begin{array}{l}3756.92 \\
3750.00 \\
3748.46 \\
3738.76 \\
3733.73\end{array}$ & $\begin{array}{r}2 \\
30 \\
15 \\
4 \\
10\end{array}$ & $\begin{array}{l}26610.01 \\
26659.12 \\
26670.07 \\
26739.26 \\
26775.28\end{array}$ & 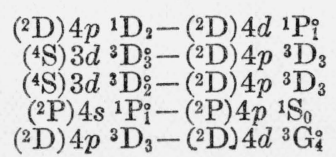 \\
\hline $\begin{array}{l}3717.94 \\
3705.54 \\
3691.88 \\
3688.44 \\
3673.83\end{array}$ & $\begin{array}{l}15 \\
2 \\
5 b \\
15 \\
18\end{array}$ & $\begin{array}{l}26888.99 \\
26978.97 \\
27078.80 \\
27104.05 \\
27211.83\end{array}$ & $\begin{array}{l}\left.\text { ( }{ }^{2} \mathrm{D}\right) 4 p^{3} \mathrm{D}_{2}-\left({ }^{2} \mathrm{D}\right) 4 d^{3} \mathrm{G}_{3} \\
\left({ }^{2} \mathrm{P}\right) 4 p^{3} \mathrm{D}_{3}-\left({ }^{2} \mathrm{P}\right) 4 d^{3} \mathrm{P}_{2}^{2} \\
\text { ('P) } 4 p p^{3} \mathrm{D}_{3}-\left({ }^{2} \mathrm{P}\right) 4 d^{3} \mathrm{D}_{3}^{3} \\
\left({ }^{2} \mathrm{D}\right) 4 p^{1} \mathrm{P}_{1}-\left({ }^{2} \mathrm{D}\right) 4 d^{3} \mathrm{~F}_{2} \\
\left({ }^{4} \mathrm{~S}\right) 3 d^{3} \mathrm{D}_{1}^{\circ}-\left({ }^{2} \mathrm{D}\right) 4 p^{3} \mathrm{~F}_{2}\end{array}$ \\
\hline $\begin{array}{l}3669.46 \\
3669.14 \\
3668.03 \\
3659.84 \\
3658.38\end{array}$ & $\begin{array}{r}2 \\
1 \\
20 \\
18 \\
20\end{array}$ & $\begin{array}{l}27244.24 \\
27246.61 \\
27254.85 \\
27315.85 \\
27326.75\end{array}$ & $\begin{array}{l}\left({ }^{4} \mathrm{~S}\right) 3 d^{3}{ }^{3} \mathrm{D}_{3}^{\circ}-\left({ }^{2} \mathrm{D}\right) 4 p^{3} \mathrm{~F}_{2} \\
\left({ }^{2} \mathrm{D}\right) 4 p^{1} \mathrm{~F}_{3}-\left({ }^{2} \mathrm{D}\right) 4 d d^{3} \mathrm{D}_{3} \\
\left({ }^{4} \mathrm{~S}\right) 3 d^{3} \mathrm{D}_{2}^{0}-\left({ }^{2} \mathrm{D}\right) 4 p{ }^{3} \mathrm{~F}_{2} \\
\left({ }^{4} \mathrm{~S}\right) 3 d^{3} \mathrm{D}_{3}^{0}-\left({ }^{2} \mathrm{D}\right) 4 p^{3} \mathrm{~F}_{3} \\
\left({ }^{4} \mathrm{~S}\right) 3 d^{3} \mathrm{D}_{2}^{\circ}-\left({ }^{2} \mathrm{D}\right) 4 p^{3} \mathrm{~F}_{3}\end{array}$ \\
\hline $\begin{array}{l}\text { 3650. } 13 \\
3648.07 \\
3639.19 \\
3623.79 \\
3618.88\end{array}$ & $\begin{array}{r}30 \\
10 \\
18 \\
9 \\
15\end{array}$ & $\begin{array}{l}27388.51 \\
27403.97 \\
27470.84 \\
27587.58 \\
27625.00\end{array}$ & $\begin{array}{l}\left.{ }^{4} \mathrm{~S}\right) 3 d^{3} \mathrm{D}_{3}-\left({ }^{2} \mathrm{D}\right) 4 p^{3} \mathrm{~F}_{4} \\
\left({ }^{2} \mathrm{D}\right) 4 p^{3} \mathrm{P}_{0}-\left({ }^{2} \mathrm{D}\right) 4 d^{3} \mathrm{~S}_{1} \\
\left({ }^{2} \mathrm{D}\right) 4 p^{3} \mathrm{P}_{1}-\left({ }^{2} \mathrm{D}\right) 4 d^{3} \mathrm{~S}_{1} \\
\left({ }^{2} \mathrm{P}\right) 4 p^{3} \mathrm{~S}_{1}-\left({ }^{2} \mathrm{P}\right) 4 d^{3} \mathrm{P}_{2}^{0} \\
\left({ }^{2} \mathrm{D}\right) 4 p^{3} \mathrm{P}_{2}-\left({ }^{2} \mathrm{D}\right) 4 d^{3} \mathrm{~S}_{1}^{\circ}\end{array}$ \\
\hline $\begin{array}{l}3615.09 \\
3610.07 \\
3609.75 \\
3605.61 \\
3605.39\end{array}$ & $\begin{array}{r}10 \\
12 \\
4 \\
7 \\
5\end{array}$ & $\begin{array}{l}27653.97 \\
27692.42 \\
27694.88 \\
27726.28 \\
27728.37\end{array}$ & 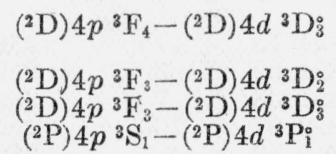 \\
\hline
\end{tabular}


TABLE 1.-Wave lengths in the second spectrum of chlorine-Continued

\begin{tabular}{|c|c|c|c|}
\hline$\lambda_{\mathrm{Bir}} \mathrm{A}$ & Intensity & $\nu_{\mathrm{vac}} \mathrm{cm}^{-1}$ & Term combination \\
\hline $\begin{array}{l}\text { 3604. } 92 \\
3604.51 \\
3603.72 \\
3600.42 \\
3595.82\end{array}$ & $\begin{array}{r}3 \\
15 \\
10 \\
5 \\
8\end{array}$ & $\begin{array}{l}27731.98 \\
27735.14 \\
27741.22 \\
27766.64 \\
27802.16\end{array}$ & $\begin{array}{l}\text { (2D) } 4 p^{3}{ }^{3} \mathrm{~F}_{2}-\left({ }^{2} \mathrm{D}\right) 4 d^{3} \mathrm{D}_{1} \\
\left({ }^{2} \mathrm{D}\right) 4 p^{3} \mathrm{P}_{0}-\left({ }^{2} \mathrm{D}\right) 4 d^{3} \mathrm{P}_{\mathrm{i}} \\
\left({ }^{2} \mathrm{D}\right) 4 p^{3} \mathrm{P}_{1}-\left({ }^{2} \mathrm{D}\right) 4 d^{3} \mathrm{P}_{0} \\
\left({ }^{2} \mathrm{D}\right) 4 p^{3} \mathrm{~F}_{2}-\left({ }^{2} \mathrm{D}\right) 4 d^{3} \mathrm{D}_{2} \\
\left({ }^{2} \mathrm{D}\right) 4 p^{3} \mathrm{P}_{1}-\left({ }^{2} \mathrm{D}\right) 4 d^{3} \mathrm{P}_{\mathrm{i}}\end{array}$ \\
\hline $\begin{array}{l}3587.78 \\
3576.00 \\
3568.04 \\
3526.13 \\
3522.14\end{array}$ & $\begin{array}{l}12 \\
15 \\
20 \\
30 \\
40\end{array}$ & $\begin{array}{l}27864.49 \\
27956.25 \\
28018.62 \\
28351.63 \\
28383.74\end{array}$ & 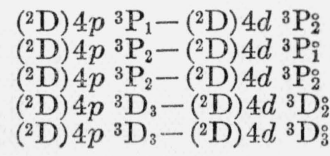 \\
\hline $\begin{array}{l}3518.28 \\
3513.69 \\
3513.22 \\
3509.39 \\
3508.94\end{array}$ & $\begin{array}{r}1 \\
12 \\
35 \\
40 \\
12\end{array}$ & $\begin{array}{l}28414.88 \\
28452.00 \\
28455.81 \\
28486.86 \\
28490.51\end{array}$ & $\begin{array}{l}\left.\text { ( }{ }^{4} \mathrm{~S}\right) 4 p^{5}{ }^{5} \mathrm{P}_{2}-4 p^{\prime}{ }^{3} \mathrm{P}_{2}^{\circ} \\
\left({ }^{2} \mathrm{D}\right) 4 p^{3} \mathrm{D}_{2}-\left({ }^{2} \mathrm{D}\right) 4 d^{3} \mathrm{D}_{1}^{\circ} \\
\left({ }^{2} \mathrm{D}\right) 4 p^{3} \mathrm{D}_{1}-\left({ }^{2} \mathrm{D}\right) 4 d^{3} \mathrm{D}_{1}^{\circ} \\
\left({ }^{2} \mathrm{D}\right) 4 p^{3} \mathrm{D}_{2}-\left({ }^{2} \mathrm{D}\right) 4 d^{3} \mathrm{D}_{2}^{\circ} \\
\left({ }^{2} \mathrm{D}\right) 4 p^{3} \mathrm{D}_{1}-\left({ }^{2} \mathrm{D}\right) 4 d^{3} \mathrm{D}_{2}^{\circ}\end{array}$ \\
\hline $\begin{array}{l}3505.44 \\
3479.82 \\
3470.40 \\
3448.14 \\
3420.36\end{array}$ & $\begin{array}{r}12 \\
30 \\
3 \\
4 \\
3\end{array}$ & $\begin{array}{l}28518.96 \\
28728.93 \\
28806.90 \\
28992.86 \\
29228.33\end{array}$ & $\left({ }^{2} \mathrm{D}\right) 4 p^{3} \mathrm{D}_{2}-\left({ }^{2} \mathrm{D}\right) 4 d^{3} \mathrm{D}_{3}^{\circ}$ \\
\hline $\begin{array}{l}3415.57 \\
3409.92 \\
3405.89 \\
3353.39 \\
3350.07\end{array}$ & $\begin{array}{r}2 \\
5 \\
3 \\
125 \\
4\end{array}$ & $\begin{array}{l}29269.32 \\
29317.82 \\
29352.51 \\
29812.03 \\
29841.58\end{array}$ & $\begin{array}{r}\left.{ }^{2} \mathrm{D}\right) 4 p^{1} \mathrm{P}_{1}-\left({ }^{2} \mathrm{D}\right) 4 d^{3} \mathrm{D}_{1} \\
\left({ }^{2} \mathrm{D}\right) 4 p^{1} \mathrm{P}_{1}-\left({ }^{2} \mathrm{D}\right) 4 d^{3} \mathrm{D}_{2}^{2} \\
3 p^{\prime}{ }^{1} \mathrm{P}_{1}^{\circ}-\left({ }^{2} \mathrm{D}\right) 4 p^{1} \mathrm{P}_{1}\end{array}$ \\
\hline $\begin{array}{l}3337.20 \\
3333.64 \\
3332.42 \\
3329.12 \\
3324.88\end{array}$ & $\begin{array}{r}3 \\
40 \\
15 \\
150 \\
1\end{array}$ & $\begin{array}{l}29956.66 \\
29988.65 \\
29999.63 \\
30029.36 \\
30067.65\end{array}$ & $\begin{array}{l}\left({ }^{4} \mathrm{~S}\right) 3 d^{3}{ }^{3} \mathrm{D}_{1}-\left({ }^{2} \mathrm{D}\right) 4 p^{3} \mathrm{P}_{2} \\
\left({ }^{4} \mathrm{~S}\right) 3 d^{3} \mathrm{D}_{2}-\left({ }^{2} \mathrm{D}\right) 4 p^{3} \mathrm{P}_{2} \\
\left({ }^{4} \mathrm{~S}\right) 3 d^{3}{ }^{3} \mathrm{D}_{2}-\left({ }^{2} \mathrm{D}\right) 4 p{ }^{3} \mathrm{P}_{2} \\
\left({ }^{4} \mathrm{~S}\right) 4 p^{3} \mathrm{P}_{2}-\left({ }^{4} \mathrm{~S}\right) 4 d^{3}{ }^{3} \mathrm{D}_{3} \\
\left({ }^{2} \mathrm{D}\right) 4 p^{3} \mathrm{P}_{2}-\left({ }^{2} \mathrm{D}\right) 4 d^{1} \mathrm{P}_{1}^{\circ}\end{array}$ \\
\hline $\begin{array}{l}3320.14 \\
3316.86\end{array}$ & $\begin{array}{l}30 \\
50\end{array}$ & $\begin{array}{l}30110.58 \\
30140.35\end{array}$ & $\begin{array}{l}\left({ }^{4} \mathrm{~S}\right) 3 d^{3} \mathrm{D}_{1}-\left({ }^{2} \mathrm{D}\right) 4 p^{3} \mathrm{P}_{1} \\
\left({ }^{4} \mathrm{~S}\right) 4 p^{3} \mathrm{P}_{2}-\left({ }^{4} \mathrm{~S}\right) 4 d^{3} \mathrm{D}_{2}^{8}\end{array}$ \\
\hline 3315.44 & 100 & 30153.26 & $\begin{array}{l}\left({ }^{4} \mathrm{~S}\right) 4 p^{3} \mathrm{P}_{1}-\left({ }^{4} \mathrm{~S}\right) 4 d^{3} \mathrm{D}_{2}^{\circ} \\
\left({ }^{4} \mathrm{~S}\right) 3 d^{3} \mathrm{D}_{2}^{0}-\left({ }^{2} \mathrm{D}\right) 4 p^{3} \mathrm{P}_{1}\end{array}$ \\
\hline $\begin{array}{l}3312.78 \\
3307.90\end{array}$ & $\begin{array}{l}15 \\
50\end{array}$ & $\begin{array}{l}30177.47 \\
30221.99\end{array}$ & $\begin{array}{l}\left.{ }^{4} \mathrm{~S}\right) 3 d^{3} \mathrm{D}_{\mathrm{i}}-\left({ }^{2} \mathrm{D}\right) 4 p^{3} \mathrm{P}_{0} \\
\left.{ }^{4} \mathrm{~S}\right) 4 p^{3} \mathrm{P}_{0}-\left({ }^{4} \mathrm{~S}\right) 4 d^{3} \mathrm{D}_{\mathrm{i}}^{\circ}\end{array}$ \\
\hline $\begin{array}{l}3306.45 \\
3276.81 \\
3231.75 \\
3222.55 \\
3203.05\end{array}$ & $\begin{array}{r}40 \\
40 \\
12 \\
7 \\
20\end{array}$ & $\begin{array}{l}\text { 30235. } 24 \\
30508.72 \\
30934.09 \\
31022.41 \\
31211.25\end{array}$ & $\begin{array}{l}\left({ }^{4} \mathrm{~S}\right) 4 p^{3} \mathrm{P}_{1}-\left({ }^{4} \mathrm{~S}\right) 4 d^{3} \mathrm{D}_{1}^{\circ} \\
\left({ }^{2} \mathrm{D}\right) 4 s^{1} \mathrm{D}_{2}-\left({ }^{2} \mathrm{P}\right) 4 p^{1} \mathrm{D}_{2} \\
\left({ }^{2} \mathrm{D}\right) 4 p^{1} \mathrm{~F}_{3}-\left({ }^{2} \mathrm{D}\right) 4 d^{1} \mathrm{D}_{2}^{\circ}\end{array}$ \\
\hline $\begin{array}{l}3202.12 \\
3189.04 \\
3187.42 \\
3181.70 \\
3181.26\end{array}$ & $\begin{array}{r}6 \\
20 \\
5 \\
7 \\
5\end{array}$ & $\begin{array}{l}31220.32 \\
31348.36 \\
31364.30 \\
31420.68 \\
31425.03\end{array}$ & $\begin{array}{l}\text { ('D) } 4 p^{3}{ }^{3} \mathrm{D}_{3}-\left({ }^{2} \mathrm{D}\right) 4 d d^{3} \mathrm{P}_{2} \\
\left({ }^{2} \mathrm{D}\right) 4 p^{3} \mathrm{D}_{1}-\left({ }^{2} \mathrm{D}\right) 4 d{ }^{3} \mathrm{P}_{0} \\
\left({ }^{2} \mathrm{D}\right) 4 p^{3} \mathrm{D}_{2}-\left({ }^{2} \mathrm{D}\right) 4 d d^{3} \mathrm{P}_{\mathrm{i}} \\
\left({ }^{2} \mathrm{D}\right) 4 p^{3} \mathrm{D}_{1}-\left({ }^{2} \mathrm{D}\right) 4 d^{3} \mathrm{P}_{\mathrm{i}}\end{array}$ \\
\hline $\begin{array}{l}3180.43 \\
3176.95 \\
3175.30 \\
3173.66 \\
3172.56\end{array}$ & $\begin{array}{r}7 \\
5 \\
6 \\
20 \\
6\end{array}$ & $\begin{array}{l}31433.23 \\
31467.66 \\
31484.01 \\
31500.28 \\
31511.20\end{array}$ & $\left({ }^{2} \mathrm{D}\right) 4 p{ }^{3} \mathrm{D}_{2}-\left({ }^{2} \mathrm{D}\right) 4 d{ }^{3} \mathrm{P}_{2}^{\circ}$ \\
\hline
\end{tabular}


TABLE 1.-Wave lengths in the second spectrum of chlorine-Continued

\begin{tabular}{|c|c|c|c|}
\hline$\lambda_{\mathrm{eir}} \mathrm{A}$ & Intensity & $\nu_{\mathrm{va} a} \mathrm{~cm}^{-1}$ & Term combination \\
\hline $\begin{array}{l}3170.23 \\
3169.45 \\
3161.44 \\
3160.52 \\
3147.86\end{array}$ & $\begin{array}{r}15 \\
7 \\
20 \\
10 \\
20\end{array}$ & $\begin{array}{l}31534.36 \\
31542.12 \\
31622.03 \\
31631.24 \\
31758.44\end{array}$ & $\begin{array}{l}\left({ }^{2} \mathrm{D}\right) 3 d^{1} \mathrm{~F}_{3}-\left({ }^{2} \mathrm{D}\right) 4 p^{1} \mathrm{D}_{2} \\
\left({ }^{2} \mathrm{D}\right) 3 d^{1} \mathrm{D}_{2}^{\circ}-\left({ }^{2} \mathrm{D}\right) 4 p^{1} \mathrm{D}_{2}\end{array}$ \\
\hline $\begin{array}{l}\text { 3125. } 96 \\
3125.44 \\
3124.28 \\
3123.72 \\
3121.62\end{array}$ & $\begin{array}{r}5 \\
6 \\
6 \\
15 \\
10\end{array}$ & $\begin{array}{l}31980.93 \\
31986.26 \\
31998.13 \\
32003.86 \\
32025.39\end{array}$ & $\begin{array}{l}\text { (2D) } 4 s^{3} \mathrm{D}_{2}^{\circ}-\left({ }^{2} \mathrm{P}\right) 4 p^{3} \mathrm{D}_{1} \\
\text { (2D) } 4 s^{3} \mathrm{D}_{3}^{3}-(2 \mathrm{P}) 4 p^{3} \mathrm{D}_{2} \\
\text { (2D) } 4 s^{3} \mathrm{D}_{1}^{\circ}-(2 \mathrm{P}) 4 p^{3} \mathrm{D}_{1} \\
\text { (2D) } 4 s^{3} \mathrm{D}_{3}^{\circ}-(2 \mathrm{P}) 4 p^{3} \mathrm{D}_{3} \\
\text { (2D) } 4 s^{3} \mathrm{D}_{2}^{\circ}-(2 \mathrm{P}) 4 p^{3} \mathrm{D}_{2}\end{array}$ \\
\hline 3119.82 & 12 & 32043.87 & $\begin{array}{l}\text { (2D) } 4 s^{3} \mathrm{D}_{2}^{2}-\left({ }^{2} \mathrm{P}\right) 4 p^{3} \mathrm{D}_{3} \\
\text { (2D) } 4 s^{3} \mathrm{D}_{1}-\left({ }^{2} \mathrm{P}\right) 4 p^{3} \mathrm{D}_{2}\end{array}$ \\
\hline $\begin{array}{l}3096.72 \\
3092.90 \\
3092.22 \\
3071.35\end{array}$ & $\begin{array}{r}25 \\
8 \\
50 \\
40\end{array}$ & $\begin{array}{l}32282.9 \\
32322.8 \\
32329.9 \\
32549.5\end{array}$ & $\begin{array}{l}\text { (3D) } 4 s^{1} \mathrm{D}_{2}^{\circ}-\left({ }^{2} \mathrm{P}\right) 4 p^{1} \mathrm{P}_{1} \\
\text { (2D) } 3 d^{3} \mathrm{~F}_{4}-(2 \mathrm{P}) 4 p^{3} \mathrm{D}_{3} \\
\text { (2D) } 3 d^{3} \mathrm{~F}_{3}^{\circ}-\left({ }^{2} \mathrm{P}\right) 4 p^{3} \mathrm{D}_{2}\end{array}$ \\
\hline $\begin{array}{l}\text { 3069. } 66 \\
3058.00 \\
3053.74 \\
3045.00 \\
3042.29\end{array}$ & $\begin{array}{r}5 \\
40 \\
10 \\
10 \\
2\end{array}$ & $\begin{array}{l}32567.4 \\
32691.6 \\
32737.2 \\
32831.2 \\
32860.4\end{array}$ & $\begin{array}{l}\text { (2D) } 3 d^{3} \mathrm{~F}_{3}^{3}-\left({ }^{2} \mathrm{P}\right) 4 p^{3} \mathrm{D}_{3} \\
\text { (2D) } 3 d^{3} \mathrm{~F}_{2}-(2 \mathrm{P}) 4 p^{3} \mathrm{D}_{1} \\
\text { (2D) } 3 d^{3} \mathrm{~F}_{2}^{\circ}-(2 \mathrm{P}) 4 p^{3} \mathrm{D}_{2} \\
\text { (2D) } 4 s^{3} \mathrm{D}_{2}^{2}-(2 \mathrm{P}) 4 p{ }^{1} \mathrm{D}_{2}\end{array}$ \\
\hline $\begin{array}{l}3037.98 \\
3036.40\end{array}$ & $\begin{array}{r}35 \\
3\end{array}$ & $\begin{array}{l}32907.1 \\
32924.2\end{array}$ & $\left({ }^{2} \mathrm{P}\right) 3 d^{3} \mathrm{D}_{3}^{\circ}-x^{\prime \prime}$ \\
\hline $\begin{array}{l}\text { 3022. } 93 \\
3018.82 \\
3006.98\end{array}$ & $\begin{array}{l}30 \\
12 \\
20\end{array}$ & $\begin{array}{l}33070.9 \\
33115.9 \\
33246.3\end{array}$ & $\begin{array}{l}\text { (2D) } 4 p p^{1} \mathrm{P}_{1}-\left({ }^{2} \mathrm{D}\right) 4 d^{1} \mathrm{D}_{2} \\
\left({ }^{2} \mathrm{D}\right) 4 s^{3} \mathrm{D}_{1}^{\circ}-4 s^{\prime}{ }^{3} \mathrm{P}_{0} \\
\left({ }^{2} \mathrm{P}\right) 3 d^{3} \mathrm{D}_{2}^{\circ}-x^{\prime \prime}\end{array}$ \\
\hline $\begin{array}{l}\text { 3006. } 05 \\
3004.39 \\
2996.63 \\
2993.09 \\
2982.78\end{array}$ & $\begin{array}{r}20 \\
10 \\
40 \\
8 \\
18\end{array}$ & $\begin{array}{l}33256.6 \\
33275.0 \\
33361.1 \\
33400.6 \\
33516.0\end{array}$ & 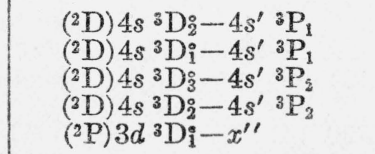 \\
\hline $\begin{array}{l}2980.90 \\
2980.47 \\
2978.48 \\
2973.46 \\
2972.63\end{array}$ & $\begin{array}{l}4 \\
2 \\
7 \\
2 \\
5\end{array}$ & $\begin{array}{l}\text { 33537. } 2 \\
33542.0 \\
33564.4 \\
33621.1 \\
33630.4\end{array}$ & $\begin{array}{l}\text { (2D) } 4 p^{3} \mathrm{D}_{1}-\left({ }^{2} \mathrm{D}\right) 4 d^{1} \mathrm{P}_{1} \\
\left({ }^{2} \mathrm{D}\right) 3 d^{3} \mathrm{~F}_{2}^{\circ}-\left({ }^{2} \mathrm{P}\right) 3 p^{1} \mathrm{D}_{2} \\
\left({ }^{2} \mathrm{D}\right) 3 d^{1} \mathrm{P}_{1}-\left({ }^{2} \mathrm{P}\right) 4 p^{1} \mathrm{P}_{1}\end{array}$ \\
\hline $\begin{array}{l}\text { 2964. } 21 \\
2950.35 \\
2945.02 \\
2937.14 \\
2934.60\end{array}$ & $\begin{array}{l}2 \\
5 \\
2 \\
2 \\
5\end{array}$ & $\begin{array}{l}33726.0 \\
33884.4 \\
33945.7 \\
34036.8 \\
34066.3\end{array}$ & $\begin{array}{l}\left({ }^{4} \mathrm{~S}\right) 4 s^{3} \mathrm{~S}_{1}-\left({ }^{2} \mathrm{D}\right) 4 p^{2} \mathrm{D}_{2} \\
\left({ }^{2} \mathrm{D}\right) 4 p^{1} \mathrm{D}_{2}-\left({ }^{2} \mathrm{D}\right) 6 s^{1} \mathrm{D}_{3}^{\circ}\end{array}$ \\
\hline $\begin{array}{l}\text { 2912. } 06 \\
\text { 2906. } 25 \\
\text { 2902. } 45\end{array}$ & $\begin{array}{r}15 \\
20 \\
4\end{array}$ & $\begin{array}{l}34329.9 \\
34398.6 \\
34443.6\end{array}$ & (2D) $4 p{ }^{1} \mathrm{P}_{1}-\left({ }^{2} \mathrm{D}\right) 4 d^{1} \mathrm{P}_{i}$ \\
\hline $\begin{array}{l}2887.41 \\
2886.63\end{array}$ & $\begin{array}{l}4 \\
3\end{array}$ & $\begin{array}{l}34623.0 \\
34632.3\end{array}$ & $\begin{array}{r}3 p^{\prime}{ }^{3} \mathrm{P}_{1}-\left({ }^{4} \mathrm{~S}\right) 4 p^{5} \mathrm{P}_{1} \\
\left({ }^{2} \mathrm{D}\right) 4 s^{3} \mathrm{D}_{1}^{\circ}-\left({ }^{2} \mathrm{P}\right) 4 p^{1} \mathrm{P}_{1}\end{array}$ \\
\hline $\begin{array}{l}2884.01 \\
2879.84 \\
2879.50 \\
2876.42 \\
2868.41\end{array}$ & $\begin{array}{r}2 \\
3 \\
2 \\
5 \\
10\end{array}$ & $\begin{array}{l}34663.8 \\
34714.0 \\
34718.1 \\
34755.3 \\
34852.3\end{array}$ & $3 p^{\prime}{ }^{3} \mathrm{P}_{1}-\left({ }^{4} \mathrm{~S}\right) 4 p^{5} \mathrm{P}_{2}$ \\
\hline
\end{tabular}


TABLE 1.-Wave lengths in the second spectrum of chlorine-Continued

\begin{tabular}{|c|c|c|c|}
\hline$\lambda_{\mathrm{Bir}} \mathrm{A}$ & Intensity & $\nu_{\mathrm{va}_{\mathrm{o}}} \mathrm{cm}^{-1}$ & Term combination \\
\hline $\begin{array}{l}\text { 2865. } 21 \\
2863.55 \\
2862.06 \\
2860.71 \\
2844.28\end{array}$ & $\begin{array}{l}4 \\
7 \\
5 \\
5 \\
4\end{array}$ & $\begin{array}{l}34891.2 \\
34911.5 \\
34929.6 \\
34946.1 \\
35148.0\end{array}$ & $\begin{array}{l}\left({ }^{2} \mathrm{D}\right) 4 s^{3} \mathrm{D}_{2}-\left({ }^{2} \mathrm{P}\right) 4 p^{3} \mathrm{P}_{2} \\
\text { (2D) } 4 s^{3} \mathrm{D}_{2}^{\circ}-\left({ }^{2} \mathrm{P}\right) 4 p^{3} \mathrm{P}_{1} \\
\text { (2D) } 4 s^{3} \mathrm{D}_{1}^{\circ}-\left({ }^{2} \mathrm{P}\right) 4 p{ }^{3} \mathrm{P}_{1} \\
\text { (2D) } 4 s^{3} \mathrm{D}_{1}^{\circ}-\left({ }^{2} \mathrm{P}\right) 4 p^{3} \mathrm{P}_{0}\end{array}$ \\
\hline $\begin{array}{l}2839.06 \\
2835.59 \\
2832.33 \\
2800.27 \\
2799.60\end{array}$ & $\begin{array}{l}1 \\
3 \\
4 \\
4 \\
4\end{array}$ & $\begin{array}{l}35212.6 \\
35255.7 \\
35296.3 \\
35700.3 \\
35708.9\end{array}$ & $\begin{array}{l}3 p^{\prime}{ }^{3} \mathrm{P}_{2}-\left({ }^{4} \mathrm{~S}\right) 4 p^{5} \mathrm{P}_{1} \\
3 p^{\prime}{ }^{3} \mathrm{P}_{2}^{\circ}-\left({ }^{4} \mathrm{~S}\right) 4 p^{5} \mathrm{P}_{2}\end{array}$ \\
\hline $\begin{array}{l}2788.63 \\
2771.78 \\
2763.88 \\
2758.69 \\
2754.10\end{array}$ & $\begin{array}{r}3 \\
2 \\
10 \\
5 \\
25\end{array}$ & $\begin{array}{l}35849.4 \\
36067.3 \\
36170.4 \\
36238.4 \\
36298.8\end{array}$ & 01 \\
\hline $\begin{array}{l}2751.52 \\
2748.09 \\
2747.98 \\
2745.75 \\
2744.25\end{array}$ & $\begin{array}{l}5 \\
1 \\
2 \\
3 \\
1\end{array}$ & $\begin{array}{l}36332.8 \\
36378.2 \\
36379.6 \\
36409.2 \\
36429.1\end{array}$ & $\left({ }^{2} \mathrm{P}\right) 4 s^{3} \mathrm{P}_{2}^{\circ}-x^{\prime \prime}$ \\
\hline $\begin{array}{l}2719.85 \\
2719.61 \\
2714.38 \\
2712.77 \\
2709.82\end{array}$ & $\begin{array}{l}1 \\
4 \\
8 \\
4 \\
2\end{array}$ & $\begin{array}{l}36755.9 \\
36759.1 \\
36829.9 \\
36851.8 \\
36891.9\end{array}$ & $\begin{array}{l}\left.{ }^{4} \mathrm{~S}\right) 3 d^{5} \mathrm{D}_{2}^{\circ}-\left({ }^{2} \mathrm{D}\right) 4 p{ }^{3} \mathrm{~F}_{2} \\
\left.{ }^{4} \mathrm{~S}\right) 3 d^{5} \mathrm{D}_{3}^{0}-\left({ }^{2} \mathrm{D}\right) 4 p p^{3} \mathrm{~F}_{2} \\
\left.{ }^{4} \mathrm{~S}\right) 3 d^{5} \mathrm{D}_{3}^{\circ}-\left({ }^{2} \mathrm{D}\right) 4 p^{3} \mathrm{~F}_{3} \\
\left({ }^{2} \mathrm{D}\right) 4 p^{3} \mathrm{P}_{1}-\left({ }^{2} \mathrm{D}\right) 6 s^{3} \mathrm{D}_{1}\end{array}$ \\
\hline $\begin{array}{l}2709.60 \\
2709.03 \\
2708.60 \\
2706.76 \\
2698.94\end{array}$ & $\begin{array}{r}4 \\
10 \\
1 \\
4 \\
1\end{array}$ & $\begin{array}{l}36894.9 \\
36902.7 \\
36908.5 \\
36933.6 \\
37040.6\end{array}$ & $\begin{array}{l}\left.{ }^{4} \mathrm{~S}\right) 3 d^{5} \mathrm{D}_{4}^{\circ}-\left({ }^{2} \mathrm{D}\right) 4 p^{3} \mathrm{~F}_{4} \\
\left({ }^{2} \mathrm{D}\right) 4 p^{3} \mathrm{P}_{1}-\left({ }^{2} \mathrm{D}\right) 6 s^{3} \mathrm{D}_{2}^{\circ}\end{array}$ \\
\hline $\begin{array}{l}2698.56 \\
2695.02 \\
2694.63 \\
2693.41 \\
2689.39\end{array}$ & $\begin{array}{l}2 \\
3 \\
3 \\
1 \\
6\end{array}$ & $\begin{array}{l}37045.8 \\
37094.5 \\
37099.8 \\
37116.7 \\
37172.1\end{array}$ & $\begin{array}{l}\left({ }^{2} \mathrm{D}\right) 4 p^{3} \mathrm{P}_{2}-\left({ }^{2} \mathrm{D}\right) 6 s^{3} \mathrm{D}_{1}^{\circ} \\
\left({ }^{2} \mathrm{D}\right) 4 p^{3} \mathrm{P}_{2}-\left({ }^{2} \mathrm{D}\right) 6 s^{3} \mathrm{D}_{3}^{\circ}\end{array}$ \\
\hline $\begin{array}{l}2688.04 \\
2679.37 \\
2676.95 \\
2672.19 \\
2671.43\end{array}$ & $\begin{array}{r}150 \\
5 \\
100 \\
50 \\
6\end{array}$ & $\begin{array}{l}37190.8 \\
37311.1 \\
37344.9 \\
37411.4 \\
37422.0\end{array}$ & $\begin{array}{l}\left({ }^{4} \mathrm{~S}\right) 4 s^{3} \mathrm{~S}_{1}-\left({ }^{2} \mathrm{D}\right) 4 p^{3} \mathrm{P}_{2} \\
\left({ }^{4} \mathrm{~S}\right) 4 s^{3} \mathrm{~S}_{1}-\left({ }^{2} \mathrm{D}\right) 4 p^{3} \mathrm{P}_{1} \\
\left({ }^{4} \mathrm{~S}\right) 4 s^{3} \mathrm{~S}_{1}-\left({ }^{2} \mathrm{D}\right) 4 p p^{3} \mathrm{P}_{0} \\
3 p^{3} \mathrm{P}_{0}^{\circ}-\left({ }^{4} \mathrm{~S}\right) 4 p^{3} \mathrm{P}_{1}\end{array}$ \\
\hline 2667.36 & 40 & 37479.1 & $\begin{array}{l}\left.\text { ( }{ }^{4} \mathrm{~S}\right) 4 p^{3} \mathrm{P}_{2}-\left({ }^{4} \mathrm{~S}\right) 6 s^{3} \mathrm{~S}_{i} \\
\left({ }^{4} \mathrm{~S}\right) 4 p^{3} \mathrm{P}_{0}-\left({ }^{4} \mathrm{~S}\right) 6 s^{3} \mathrm{~S}_{1}^{\circ}\end{array}$ \\
\hline $\begin{array}{l}2666.46 \\
2659.67 \\
2658.74 \\
2648.19\end{array}$ & $\begin{array}{r}20 \\
3 \\
100 \\
10\end{array}$ & $\begin{array}{l}37491.8 \\
37587.5 \\
37600.6 \\
37750.4\end{array}$ & $\begin{array}{l}\left({ }^{4} \mathrm{~S}\right) 4 p^{3} \mathrm{P}_{1}-\left({ }^{4} \mathrm{~S}\right) 6 s^{3} \mathrm{~S}_{i} \\
3 p^{\prime}{ }^{1} \mathrm{P}_{1}-\left({ }^{2} \mathrm{D}\right) 4 p{ }^{1} \mathrm{D}_{2}\end{array}$ \\
\hline 2647. 79 & 5 & 37756.1 & $3 p^{\prime}{ }^{3} \mathrm{P}_{1}-\left({ }^{4} \mathrm{~S}\right) 4 p^{3} \mathrm{P}_{1}$ \\
\hline 2646. 88 & 25 & 37769.1 & $\begin{array}{l}3 p^{\prime}{ }^{3} \mathrm{P}_{1}-\left({ }^{4} \mathrm{~S}\right) 4 p^{3} \mathrm{P}_{2} \\
3 p^{3} \mathrm{P}_{1}-\left({ }^{4} \mathrm{~S}\right) 4 p^{3} \mathrm{P}_{0}\end{array}$ \\
\hline $\begin{array}{l}\text { 2642. } 28 \\
2635.82 \\
2635.44\end{array}$ & $\begin{array}{l}4 \\
3 \\
3\end{array}$ & $\begin{array}{l}37834.8 \\
37927.6 \\
37933.0\end{array}$ & \\
\hline
\end{tabular}


TABLE 1.-Wave lengths in the second spectrum of chlorine-Continued

\begin{tabular}{|c|c|c|c|}
\hline$\lambda_{\mathrm{air}} \mathrm{A}$ & Intensity & $\nu_{\mathrm{Vac}} \mathrm{cm}^{-1}$ & Term combination \\
\hline $\begin{array}{l}2634.95 \\
2634.10 \\
2631.33 \\
2630.20 \\
2626.91\end{array}$ & $\begin{array}{r}12 \\
2 \\
2 \\
4 \\
3\end{array}$ & $\begin{array}{l}37940.1 \\
37952.3 \\
37992.3 \\
38008.6 \\
38056.2\end{array}$ & $\begin{array}{l}\left({ }^{2} \mathrm{D}\right) 3 d^{1} \mathrm{~F}_{3}^{\circ}-\left({ }^{2} \mathrm{P}\right) 4 p^{1} \mathrm{D}_{2} \\
\left({ }^{2} \mathrm{D}\right) 3 d^{3} \mathrm{~F}_{3}^{\circ}-x^{\prime}\end{array}$ \\
\hline $\begin{array}{l}2621.87 \\
2619.80 \\
2617.91 \\
2615.13 \\
2614.65\end{array}$ & $\begin{array}{r}4 \\
4 \\
1 \\
10 \\
5\end{array}$ & $\begin{array}{l}38129.4 \\
38159.5 \\
38187.0 \\
38227.6 \\
38234.6\end{array}$ & $\begin{array}{l}\text { (2D) } 4 p^{3} \mathrm{D}_{3}-\left({ }^{2} \mathrm{P}\right) 4 d^{3} \mathrm{~F}_{4} \\
\left({ }^{2} \mathrm{D}\right) 4 p^{3} \mathrm{D}_{3}-\left({ }^{2} \mathrm{P}\right) 4 d^{3} \mathrm{~F}_{3}^{\circ}\end{array}$ \\
\hline $\begin{array}{l}2608.72 \\
2608.24 \\
2605.67 \\
2604.18 \\
2603.36\end{array}$ & $\begin{array}{r}2 \\
2 \\
5 \\
8 \\
10\end{array}$ & $\begin{array}{l}38321.5 \\
38328.6 \\
38366.4 \\
38388.3 \\
38400.4\end{array}$ & $\begin{array}{c}\left({ }^{2} \mathrm{D}\right) 4 p^{3} \mathrm{D}_{2}-\left({ }^{2} \mathrm{P}\right) 4 d^{3} \mathrm{~F}_{3}^{\circ} \\
\left({ }^{2} \mathrm{D}\right) 4 p^{3} \mathrm{D}_{1}-\left({ }^{2} \mathrm{P}\right) 4 d^{3} \mathrm{~F}_{2}^{0} \\
\\
3 p^{\prime}{ }^{3} \mathrm{P}_{2}-\left({ }^{4} \mathrm{~S}\right) 4 p^{3} \mathrm{P}_{1} \\
3 p^{\prime}{ }^{3} \mathrm{P}_{2}^{0}-\left({ }^{4} \mathrm{~S}\right) 4 p{ }^{3} \mathrm{P}_{2}\end{array}$ \\
\hline $\begin{array}{l}2582.82 \\
2580.40 \\
2571.10 \\
2568.25 \\
2568.13\end{array}$ & $\begin{array}{l}3 \\
4 \\
8 \\
3 \\
4\end{array}$ & $\begin{array}{l}38705.8 \\
38742.1 \\
38882.2 \\
38925.4 \\
38927.2\end{array}$ & 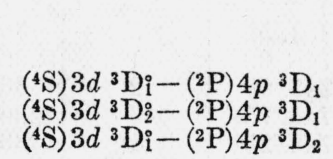 \\
\hline $\begin{array}{l}2566.01 \\
2565.29 \\
2564.84 \\
2564.13 \\
2549.85\end{array}$ & $\begin{array}{r}5 \\
15 \\
20 \\
6 \\
50\end{array}$ & $\begin{array}{l}\text { 38959. } 4 \\
38970.3 \\
38977.1 \\
38987.9 \\
39206.2\end{array}$ & $\begin{array}{l}\left({ }^{4} \mathrm{~S}\right) 3 d^{3} \mathrm{D}_{3}^{\circ}-\left({ }^{2} \mathrm{P}\right) 4 p{ }^{3} \mathrm{D}_{2} \\
\left({ }^{4} \mathrm{~S}\right) 3 d^{3} \mathrm{D}_{2}^{\circ}-\left({ }^{2} \mathrm{P}\right) 4 p^{3} \mathrm{D}_{2} \\
\left({ }^{4} \mathrm{~S}\right) 3 d^{3} \mathrm{D}_{3}^{3}-\left({ }^{2} \mathrm{P}\right) 4 p p^{3} \mathrm{D}_{3} \\
\left({ }^{4} \mathrm{~S}\right) 3 d^{3} \mathrm{D}_{2}^{\circ}-\left({ }^{2} \mathrm{P}\right) 4 p p^{3} \mathrm{D}_{3} \\
\left.{ }^{4} \mathrm{~S}\right) 4 p^{3} \mathrm{P}_{2}-\left({ }^{4} \mathrm{~S}\right) 5 d^{3} \mathrm{D}_{3}^{3}\end{array}$ \\
\hline $\begin{array}{l}2547.76 \\
2546.94 \\
2544.84 \\
2543.98 \\
2520.09\end{array}$ & $\begin{array}{r}12 \\
20 \\
15 \\
10 \\
2\end{array}$ & $\begin{array}{l}\text { 39238. } 4 \\
39251.0 \\
39283.4 \\
39296.7 \\
39669.2\end{array}$ & 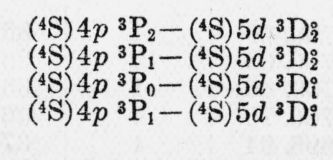 \\
\hline $\begin{array}{l}2518.15 \\
2515.92 \\
2514.01 \\
2513.34 \\
2512.41\end{array}$ & $\begin{array}{l}4 \\
3 \\
3 \\
1 \\
2\end{array}$ & $\begin{array}{l}\text { 39699. } 8 \\
39734.9 \\
39765.1 \\
39775.7 \\
39790.4\end{array}$ & $\begin{array}{l}\left({ }^{2} \mathrm{D}\right) 4 p{ }^{3} \mathrm{~F}_{4}-\left({ }^{2} \mathrm{D}\right) 6 s^{3} \mathrm{D}_{3}^{\circ} \\
\left({ }^{2} \mathrm{D}\right) 4 p{ }^{3} \mathrm{~F}_{3}-\left({ }^{2} \mathrm{D}\right) 6 s^{3} \mathrm{D}_{2} \\
\left.{ }^{4} \mathrm{~S}\right) 3 d^{3} \mathrm{D}_{3}^{3}-\left({ }^{2} \mathrm{P}\right) 4 p{ }^{1} \mathrm{D}_{2} \\
\left.{ }^{4} \mathrm{~S}\right) 3 d^{3} \mathrm{D}_{2}-\left({ }^{2} \mathrm{P}\right) 4 p{ }^{1} \mathrm{D}_{2} \\
\left({ }^{2} \mathrm{D}\right) 4 p^{3} \mathrm{~F}_{2}-\left({ }^{2} \mathrm{D}\right) 6 s^{3} \mathrm{D}_{1}^{\circ}\end{array}$ \\
\hline $\begin{array}{l}2511.33 \\
2502.75 \\
2498.53 \\
2496.04 \\
2492.84\end{array}$ & $\begin{array}{r}3 \\
40 \\
30 \\
20 \\
3\end{array}$ & $\begin{array}{l}39807.6 \\
39944.0 \\
40011.5 \\
40051.4 \\
40102.8\end{array}$ & $\begin{array}{l}\left({ }^{2} \mathrm{D}\right) 4 p{ }^{3} \mathrm{~F}_{2}-\left({ }^{2} \mathrm{D}\right) 6 s^{3} \mathrm{D}_{2}^{\circ} \\
\left({ }^{4} \mathrm{~S}\right) 4 p \\
{ }^{5} \mathrm{P}_{3}-\left({ }^{4} \mathrm{~S}\right) 6 s^{5} \mathrm{~S}_{2}^{\circ} \\
\left({ }^{4} \mathrm{~S}\right) 4 p{ }^{5} \mathrm{P}_{2}-\left({ }^{4} \mathrm{~S}\right) 6 s^{5} \mathrm{~S}_{2} \\
\left({ }^{4} \mathrm{~S}\right) 4 p{ }^{5} \mathrm{P}_{1}-\left({ }^{4} \mathrm{~S}\right) 6 s^{5} \mathrm{~S}_{2}^{\circ}\end{array}$ \\
\hline $\begin{array}{l}2492.65 \\
2472.69 \\
2467.50 \\
2466.72 \\
2459.86\end{array}$ & $\begin{array}{r}2 \\
3 \\
1 \\
2 \\
10\end{array}$ & $\begin{array}{l}\text { 40105. } 8 \\
40429.6 \\
40514.6 \\
40527.4 \\
40640.4\end{array}$ & $\begin{array}{l}\text { (2D) } 4 p^{3} \mathrm{D}_{3}-\left({ }^{2} \mathrm{D}\right) 6 s^{3} \mathrm{D}_{3}^{3} \\
\text { (2D) } 4 p^{3} \mathrm{D}_{1}-(2 \mathrm{D}) 6 s^{3} \mathrm{D}_{1} \\
\text { (2D) } 4 p^{3} \mathrm{D}_{2}-\left({ }^{2} \mathrm{D}\right) 6 s^{3} \mathrm{D}_{2}^{\circ}\end{array}$ \\
\hline $\begin{array}{l}2452.30 \\
2445.34 \\
2444.12 \\
2440.98 \\
2440.49\end{array}$ & $\begin{array}{r}10 \\
20 \\
7 \\
3 \\
4\end{array}$ & $\begin{array}{l}\text { 40765. } 7 \\
\text { 40881. } 7 \\
40902.1 \\
\text { 40954. } 7 \\
\text { 40963. } 0\end{array}$ & \\
\hline
\end{tabular}


TABLE 1.-Wave lengths in the second spectrum of chlorine-Continued

\begin{tabular}{|c|c|c|c|}
\hline$\lambda_{\mathrm{air}} \mathrm{A}$ & Intensity & $\nu_{\mathrm{va}} \mathrm{cm}^{-1}$ & Term combination \\
\hline 2440. 33 & 5 & 40965. 6 & \\
\hline 2434. 10 & 50 & 41070.5 & 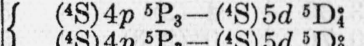 \\
\hline 2433. 26 & 3 & 41084. 7 & \\
\hline 2430.16 & 30 & 41137. 1 & $\left\{\begin{array}{l}\left.{ }^{(4} \mathrm{S}\right) 4 p^{5} \mathrm{P}_{2}-\left({ }^{4} \mathrm{~S}\right) 5 d^{5} \mathrm{D}_{3}^{0} \\
\left.{ }^{4} \mathrm{~S}\right) 4 p^{5} \mathrm{P}_{4}-\left({ }^{4} \mathrm{~S}\right) 5 d^{5} \mathrm{D}^{0}\end{array}\right.$ \\
\hline 2428.02 & 10 & 41173. 3 & \\
\hline $\begin{array}{l}2427.79 \\
2424.01 \\
2420.30 \\
2419.85 \\
2412.48\end{array}$ & $\begin{array}{r}20 \\
10 \\
2 \\
4 \\
10\end{array}$ & $\begin{array}{l}41177.2 \\
41241.4 \\
41304.6 \\
41312.3 \\
41438.5\end{array}$ & $\left({ }^{4} \mathrm{~S}\right) 4 p^{5} \mathrm{P}_{1}-\left({ }^{4} \mathrm{~S}\right) 5 d{ }^{5} \mathrm{D}_{2}^{*}$ \\
\hline $\begin{array}{l}2407.10 \\
2405.86 \\
2405.21 \\
2404.59 \\
2404.15\end{array}$ & $\begin{array}{l}5 \\
2 \\
1 \\
5 \\
2\end{array}$ & $\begin{array}{l}41531.1 \\
41552.5 \\
41563.8 \\
41574.5 \\
41582.1\end{array}$ & \\
\hline $\begin{array}{l}2403.87 \\
2401.87 \\
2400.62 \\
2399.85 \\
2398.91\end{array}$ & $\begin{array}{l}3 \\
2 \\
1 \\
3 \\
2\end{array}$ & $\begin{array}{l}41586.9 \\
41621.6 \\
41643.2 \\
41656.6 \\
41672.9\end{array}$ & ( $\left.{ }^{2} \mathrm{D}\right) 4 p^{1} \mathrm{P}_{1}-\left({ }^{2} \mathrm{D}\right) 6 s^{1} \mathrm{D}_{3}$ \\
\hline $\begin{array}{l}\text { 2397. } 81 \\
2380.46 \\
2365.80 \\
2340.60 \\
2332.90\end{array}$ & $\begin{array}{l}1 \\
2 \\
2 \\
2 \\
2\end{array}$ & $\begin{array}{l}\text { 41692. } 0 \\
\text { 41995. } 9 \\
\text { 42256. } 1 \\
\text { 42711. } 0 \\
42851.9\end{array}$ & $\left({ }^{2} \mathrm{D}\right) 3 d^{1} \mathrm{D}_{2}^{2}-x^{\prime}$ \\
\hline $\begin{array}{l}2327.10 \\
2323.02 \\
2322.00 \\
2321.28 \\
2320.25\end{array}$ & $\begin{array}{l}2 \\
4 \\
1 \\
1 \\
2\end{array}$ & $\begin{array}{l}42958.7 \\
43034.2 \\
43053.1 \\
43066.4 \\
43085.5\end{array}$ & $\begin{array}{l}\left.{ }^{4} \mathrm{~S}\right) 4 p{ }^{3} \mathrm{P}_{2}-\left({ }^{2} \mathrm{D}\right) 4 d^{3} \mathrm{D}_{2} \\
\left.{ }^{4} \mathrm{~S}\right) 4 p \\
{ }^{3} \mathrm{P}_{1}-\left({ }^{2} \mathrm{D}\right) 4 d^{3} \mathrm{D}_{2}^{0} \\
\left({ }^{4} \mathrm{~S}\right) 4 p \\
{ }^{3} \mathrm{P}_{2}-\left({ }^{2} \mathrm{D}\right) 4 d_{d i}^{3} \mathrm{D}_{3}^{3}\end{array}$ \\
\hline $\begin{array}{l}2308.94 \\
2304.59 \\
2295.27 \\
2288.17 \\
2276.25\end{array}$ & $\begin{array}{l}2 \\
1 \\
1 \\
7 \\
4\end{array}$ & $\begin{array}{l}43296.6 \\
43378.3 \\
43554.4 \\
43689.5 \\
43918.3\end{array}$ & \\
\hline $\begin{array}{l}2253.16 \\
2251.50 \\
2250.96 \\
2109.37 \\
2102.99\end{array}$ & $\begin{array}{r}30 \\
40 \\
20 \\
2 \\
3\end{array}$ & $\begin{array}{l}\text { 44368. } 3 \\
44401.0 \\
44411.7 \\
47392.4 \\
47536.2\end{array}$ & $\begin{array}{l}\left({ }^{4} \mathrm{~S}\right) 3 d^{3} \mathrm{D}_{\mathrm{i}}-x^{\prime} \\
\left({ }^{4} \mathrm{~S}\right) 3 d^{3} \mathrm{D}_{3}^{\circ}-x^{\prime} \\
\left({ }^{4} \mathrm{~S}\right) 3 d^{3} \mathrm{D}_{2}^{\circ}-x^{\prime} \\
\left({ }^{4} \mathrm{~S}\right) 4 s^{3} \mathrm{~S}_{\mathrm{i}}-4 s^{\prime}{ }^{3} \mathrm{P}_{1} \\
\left({ }^{4} \mathrm{~S}\right) 4 s^{3}{ }^{3} \mathrm{~S}_{1}^{\circ}-4 s^{\prime}{ }^{3} \mathrm{P}_{2}\end{array}$ \\
\hline
\end{tabular}


TABLE 2.-Classified lines of $\mathrm{Cl}_{\text {II }}$ in the Schumann region

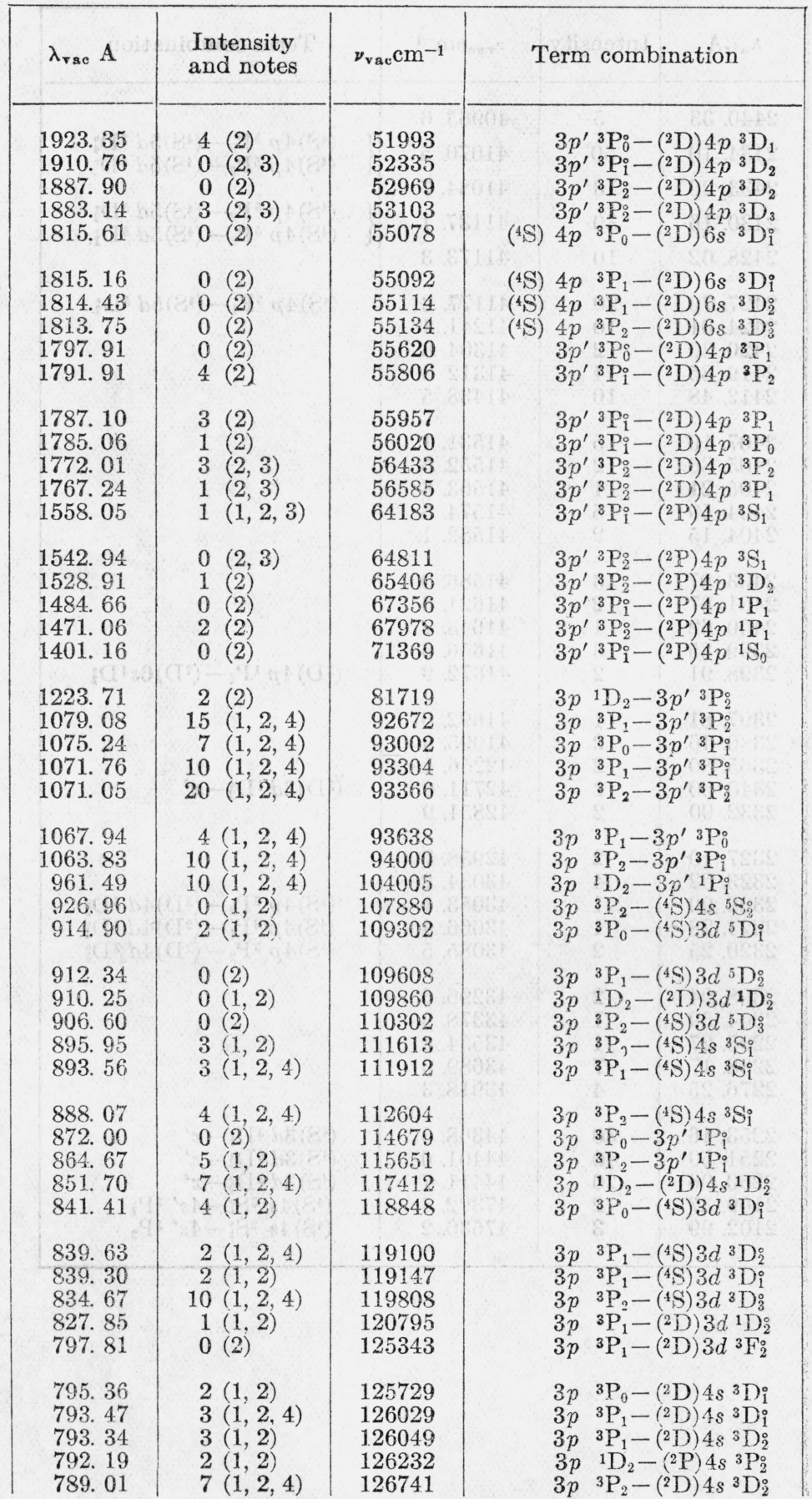


TABLE 2.-Classified lines of $\mathrm{Cl}$ II $i n$ the Schumann region-Continued

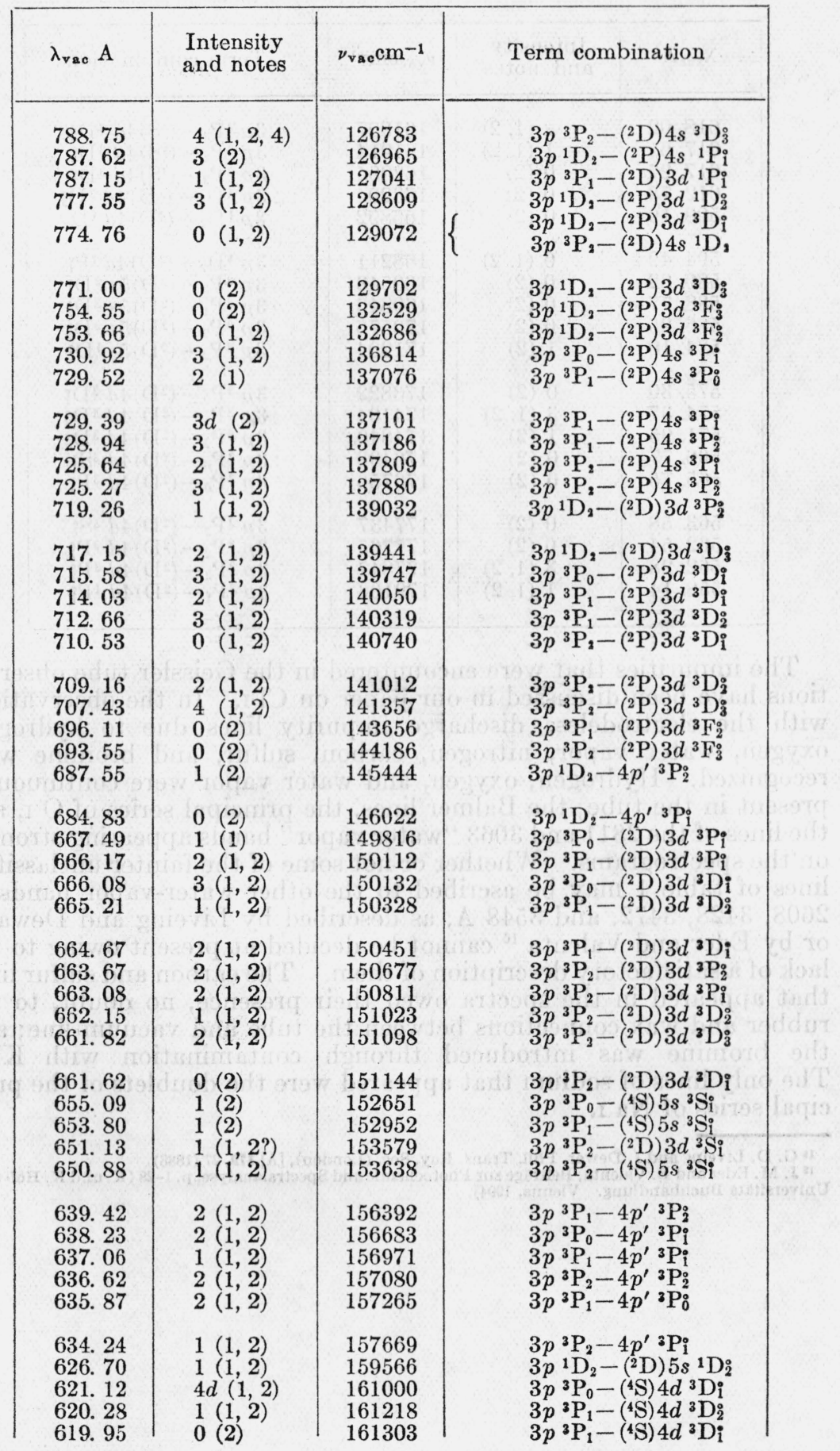


TABLE 2.-Classified lines of $\mathrm{CI}$ II in the Schumann region-Continued

\begin{tabular}{|c|c|c|c|}
\hline$\lambda_{\mathrm{Vac}} \mathrm{A}$ & $\begin{array}{l}\text { Intensity } \\
\text { and notes }\end{array}$ & $\nu_{\mathrm{vac}} \mathrm{cm}^{-1}$ & Term combination \\
\hline $\begin{array}{l}\text { 618. } 02 \\
617.61 \\
617.27 \\
612.73 \\
599.19\end{array}$ & $\begin{array}{l}2(1,2) \\
1(1,2) \\
0(2) \\
0(2) \\
0(2)\end{array}$ & $\begin{array}{l}161807 \\
161914 \\
162004 \\
163204 \\
166892\end{array}$ & 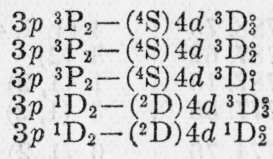 \\
\hline $\begin{array}{l}594.49 \\
589.82 \\
588.77 \\
586.25 \\
584.10\end{array}$ & $\begin{array}{l}0(1,2) \\
0(2) \\
0(2) \\
0(2) \\
1(2)\end{array}$ & $\begin{array}{l}168211 \\
169543 \\
169846 \\
170576 \\
171204\end{array}$ & $\begin{array}{l}3 p^{1} \mathrm{D}_{2}-\left({ }^{2} \mathrm{D}\right) 4 d^{1} \mathrm{P}_{1} \\
3 p^{3} \mathrm{P}_{0}-\left({ }^{2} \mathrm{D}\right) 5 s^{3} \mathrm{D}_{1}^{\circ} \\
3 p^{3} \mathrm{P}_{1}-(2 \mathrm{D}) 5 s^{3} \mathrm{D}_{2} \\
3 p^{3} \mathrm{P}_{2}-\left({ }^{2} \mathrm{D}\right) 5 s^{3} \mathrm{D}_{3}^{\circ} \\
3 p^{3} \mathrm{P}_{2}-\left({ }^{2} \mathrm{D}\right) 5 s^{1} \mathrm{D}_{2}^{\circ}\end{array}$ \\
\hline $\begin{array}{l}575.30 \\
574.37 \\
571.95 \\
566.77 \\
565.75\end{array}$ & $\begin{array}{l}0(2) \\
3(1,2) \\
1(2) \\
0(2) \\
0(2)\end{array}$ & $\begin{array}{l}173822 \\
174104 \\
174840 \\
176438 \\
176756\end{array}$ & $\begin{array}{l}3 p^{3} \mathrm{P}_{0}-\left({ }^{2} \mathrm{D}\right) 4 d^{3} \mathrm{D}_{\mathrm{i}} \\
3 p{ }^{3} \mathrm{P}_{1}-\left({ }^{2} \mathrm{D}\right) 4 d^{3} \mathrm{D}_{2}^{\circ} \\
3 p{ }^{3} \mathrm{P}_{2}-(2 \mathrm{D}) 4 d^{3} \mathrm{D}_{3}^{\circ} \\
3 p{ }^{3} \mathrm{P}_{0}-\left({ }^{2} \mathrm{D}\right) 4 d^{3} \mathrm{~S}_{\mathrm{i}} \\
3 p^{3} \mathrm{P}_{0}-\left({ }^{2} \mathrm{D}\right) 4 d^{3} \mathrm{P}_{\mathrm{i}}\end{array}$ \\
\hline $\begin{array}{l}563.58 \\
562.54 \\
562.28 \\
558.14\end{array}$ & $\begin{array}{l}0(2) \\
0(2) \\
3(1,2) \\
1(1,2)\end{array}$ & $\begin{array}{l}177437 \\
177765 \\
177847 \\
179166\end{array}$ & $\begin{array}{l}3 p^{3} \mathrm{P}_{2}-\left({ }^{2} \mathrm{D}\right) 4 d^{3} \mathrm{~S}_{\mathrm{i}}^{\circ} \\
3 p{ }^{3} \mathrm{P}_{2}-\left({ }^{2} \mathrm{D}\right) 4 d^{3} \mathrm{P}_{1} \\
3 p{ }^{3} \mathrm{P}_{2}-\left({ }^{2} \mathrm{D}\right) 4 d^{3} \mathrm{P}_{2} \\
3 p^{3} \mathrm{P}_{1}-\left({ }^{2} \mathrm{D}\right) 4 d^{1} \mathrm{P}_{\mathrm{i}}\end{array}$ \\
\hline
\end{tabular}

The impurities that were encountered in the Geissler tube observations have been discussed in our paper on $\mathrm{Cl}$ I. In the observations with the electrodeless discharge impurity lines due to hydrogen, oxygen, water vapor, nitrogen, carbon, sulfur, and bromine were recognized. Hydrogen, oxygen, and water vapor were continuously present in the tube; the Balmer lines, the principal series of $\mathrm{O}_{\mathrm{I}}$, and the lines of the 2811 and 3063 "water vapor" bands appearing strongly on the spectrograms. Whether or not some of the fainter unclassified lines of table 1 may be ascribed to the other water-vapor bands at $2608,3428,3472$, and $3548 \mathrm{~A}$, as described by Liveing and Dewar, ${ }^{16}$ or by Eder and Valenta ${ }^{16}$ cannot be decided at present owing to the lack of a satisfactory description of them. The carbon and sulfur lines that appeared in the spectra owed their presence, no doubt, to the rubber and wax connections between the tube and vacuum line; and the bromine was introduced through contamination with $\mathrm{KBr}$. The only lines of sodium that appeared were the doublets of the principal series of $\mathrm{Na}$.

1s G. D. Liveing and J. Dewar, Phil. Trans. Roy. Soc. (London), [A] 179, 27 (1888).

10 J. M. Eder and E. Valenta, Beitrüge zur Photochemie and Spectralanalyse, p. 1-28 (K. und K. Hof- un d Universitäts Buchhandlung. Vienna, 1904). 
The lines in table 2 have been obtained from different sources. As stated above, we have had at our disposal unpublished lists of observations in the extreme ultraviolet by Bowen, by Weinberg, and by Boyce. In addition to these, there is a list extending to $1300 \mathrm{~A}$ published by Vaudet. ${ }^{17}$ The wave lengths in the first column are adjusted mean values for all lines given by more than one author. The intensities in the second column are the maximum estimates given by any one of the authors. The numbers in parentheses after the intensities refer to the sources from which the data have been drawn, as follows: (1) for Bowen, (2) for Boyce, (3) for Vaudet, and (4) for Weinberg.

Except for lines indicated above as doubtful, we believe that the wave lengths recorded in tables 1 and 2 give a thorough description of the spectrum emitted by singly ionized chlorine atoms. Qualitatively there is good agreement between our list and the corrected list published by L. and E. Bloch. ${ }^{18}$ All lines common to the two lists appear in table 1, but each list contains faint lines not to be found in the other. Such lines that cannot be satisfactorily accounted for by term combinations are probably due to impurities not yet recognized.

\section{TERM STRUCTURE}

The classified lines of $\mathrm{Cl}$ Ir may be fully accounted for as combinations of the terms given in table 4. The neutral atom has 17 extranuclear electrons, of which 10 fill all the available places within the $K$ and $L$ shells, leaving 7 to be assigned to the $M$ and outer shells. In the unexcited state of the neutral atom, two of the seven valence electrons occupy $s$ orbits and five occupy $p$ orbits of the $M$ shell, the configuration being designated symbolically as $3 s^{2} 3 p^{5}$. In the process of excitation and ionization, one of the $p$ or one of the $s$ electrons may be raised to successively higher orbits until it is removed from control of the atom, leaving it ionized.

The terms that are descriptive of the energy states of the singly ionized atom when the electrons are in either of the configurations $3 s^{2} 3 p^{4}$ or $3 s 3 p^{5}$, are the limits approached by the series of Clr as the excitation of the neutral atom proceeds towards ionization. Similarly, excitation of the ion will lead to sequences of terms which approach the basic terms of Clin as limits. There will, therefore, be several families of terms of $\mathrm{Cl}$ II, each arising from adding the energy of the migrating valence electron, by the quantum methods, to that of either a basic or a higher term of ClnI, which are known from the work of

17 G. Vaudet, Compt. rend. 185, 1271 (1927).

18 L. and E. Bloch, Ann. phys. [10] 8, 397 (1927); [10] 9, 554 (1928). 
Bowen. ${ }^{19}$ The terms thus expected, theoretically, are arrayed in table 3. The terms that have actually been found are listed in table 4 , of which figure 1 is a graphical representation. The terms of $\mathrm{Cl}$ Ir

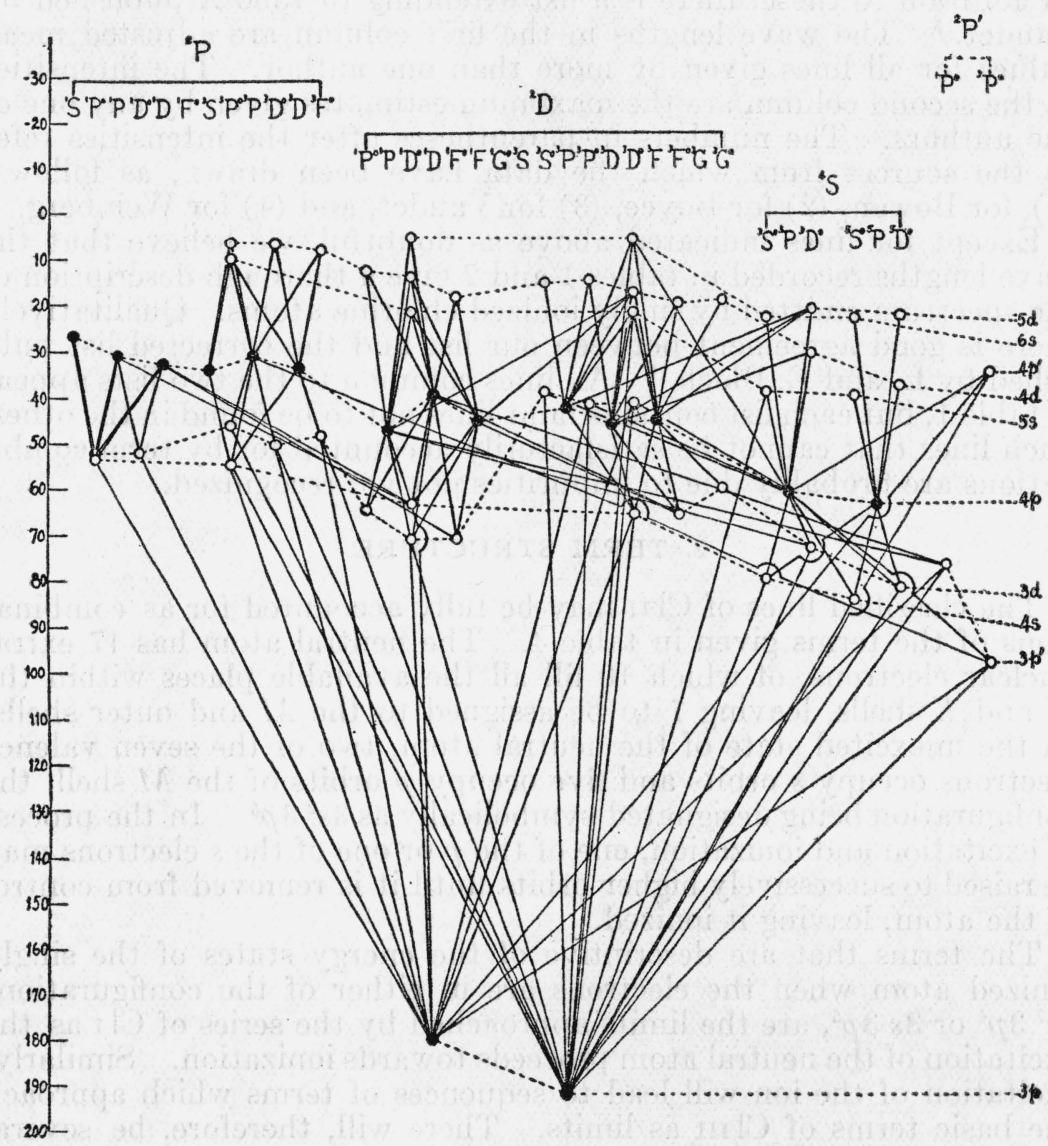

Figure 1.-Term diagram for $\mathrm{Clir}$.

indicates even terms; $O$ odd terms

are thus seen to belong to four, possibly to five, families. Of these, the terms arising from ${ }^{4} \mathrm{~S},{ }^{2} \mathrm{D}$, and ${ }^{2} \mathrm{P}$ of $\mathrm{Cl}$ III are the most conspicuous. They account for nearly all the lines of high intensity.

10 I. S. Bowen, Phys. Rev. 45, 403 (1934). 
TABLE 3.-Theoretical terms of $\mathrm{Cl} \mathrm{II}$

\begin{tabular}{|c|c|c|c|}
\hline Electron configuration & \multicolumn{3}{|c|}{ Terms } \\
\hline $\begin{array}{l}3 s^{2} 3 p^{4} \\
n s 3 p^{5}=n p^{\prime} \\
3 s 3 p^{4} n s=n s^{\prime}\end{array}$ & \multicolumn{3}{|c|}{$\begin{array}{c}{ }^{3} \mathrm{P},{ }^{1} \mathrm{D},{ }^{1} \mathrm{~S} \\
{ }^{5} \mathrm{P},{ }^{3} \mathrm{D},{ }^{3} \mathrm{P},{ }^{\circ}{ }^{\circ}{ }^{1},{ }^{\circ} \mathrm{S},{ }^{1} \mathrm{D},{ }^{1} \mathrm{P},{ }^{1} \mathrm{~S}\end{array}$} \\
\hline $\begin{array}{l}\text { Basic terms of } \\
\mathrm{Cl} \mathrm{III}\end{array}$ & \multirow[t]{2}{*}{${ }^{4} \mathrm{~S}^{\circ}$} & \multirow[t]{2}{*}{${ }^{2} \mathrm{D}^{\circ}$} & \multirow[t]{2}{*}{${ }^{2} \mathrm{P}^{\circ}$} \\
\hline $3 s^{2} 3 p^{3}$ & & & \\
\hline $\begin{array}{l}3 s^{2} 3 p^{3} n s \\
3 s^{2} 3 p^{3} n p \\
3 s^{2} 3 p^{3} n d\end{array}$ & $\begin{array}{l}{ }^{5} \mathrm{~S}^{\circ},{ }^{3} \mathrm{~S}^{\circ} \\
{ }^{5} \mathrm{P},{ }^{3} \mathrm{P} \\
{ }^{5} \mathrm{D}^{\circ},{ }^{3} \mathrm{D}^{\circ}\end{array}$ & 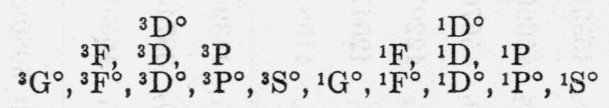 & $\begin{array}{c}{ }^{3} \mathrm{P}^{\circ} \\
{ }^{3} \mathrm{D},{ }^{3} \mathrm{P},{ }^{3} \mathrm{~S},{ }^{1} \mathrm{D},{ }^{1} \mathrm{P}, \\
{ }^{3} \mathrm{~F}^{\circ},{ }^{\circ} \mathrm{D}^{\circ},{ }^{\circ} \mathrm{P}^{\circ},{ }^{1} \mathrm{~F}^{\circ},{ }^{1} \mathrm{D}^{\circ},{ }^{1} \mathrm{P}^{\circ}\end{array}$ \\
\hline
\end{tabular}


TABLE 4.-Observed terms of $\mathrm{Cl} \mathrm{II}$

\begin{tabular}{|c|c|c|c|}
\hline & Limit: ${ }^{4} \mathrm{~S}=321936$ & $\operatorname{Limit}\left\{\begin{array}{l}{ }^{2} D_{21 / 2}=303816 \\
{ }^{2} D_{1 / 2}=303883\end{array}\right.$ & Limit $\left\{\begin{array}{l}{ }^{2} P_{1 / 2}=292029 \\
{ }^{2} P_{03 / 4}=292124\end{array}\right.$ \\
\hline $3 p$ & 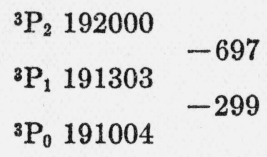 & ${ }^{1} D_{2} 180348$ & ${ }^{1} \mathrm{~S}_{0}$ \\
\hline $4 p$ & 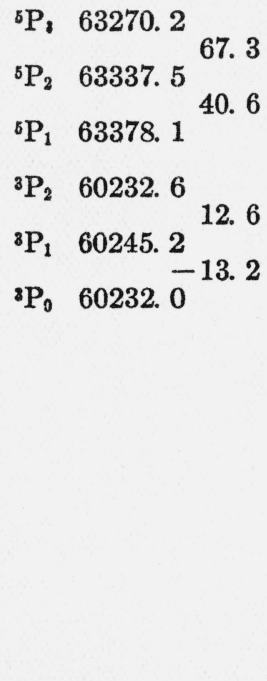 & 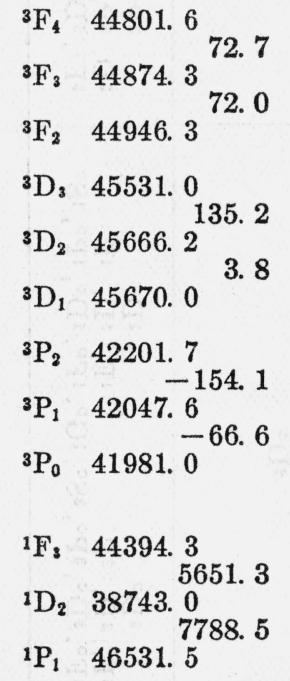 & 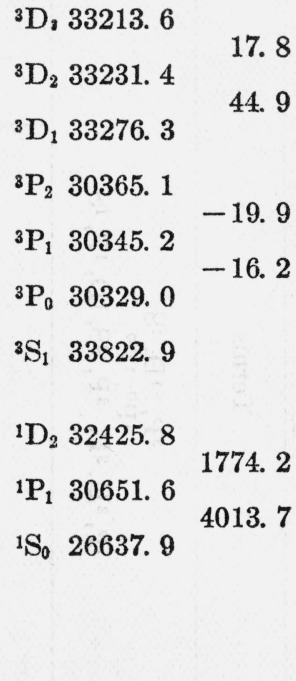 \\
\hline $3 d$ & 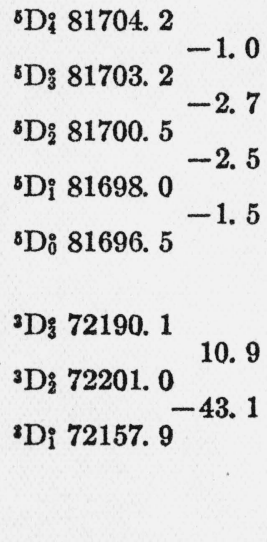 & 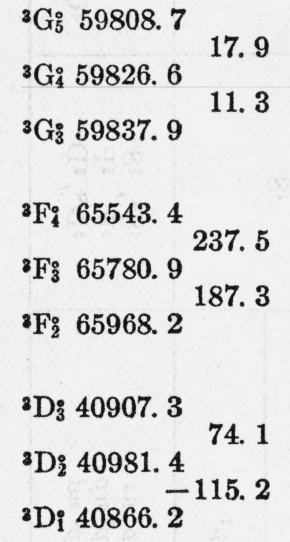 & 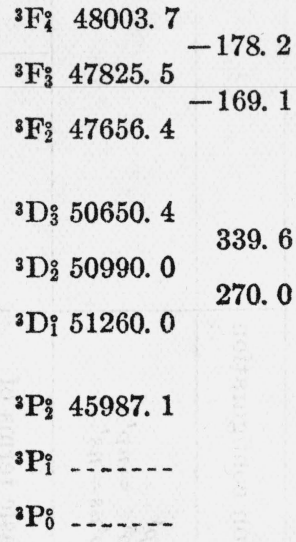 \\
\hline
\end{tabular}


TABLE 4.-Observed terms of $\mathrm{Cl} \mathrm{II}-$ Continued

\begin{tabular}{|c|c|c|c|}
\hline & Limit: ${ }^{4} \mathrm{~S}=321936$ & Limit $\left\{\begin{array}{l}{ }^{2} D_{21 / 2}=303816 \\
{ }^{2} D_{1 / 2}=303883\end{array}\right.$ & Limit $\left\{\begin{array}{l}2 P_{1 / 2}=292029 \\
{ }^{2} P_{01 / 2}=292124\end{array}\right.$ \\
\hline $3 d$ & & 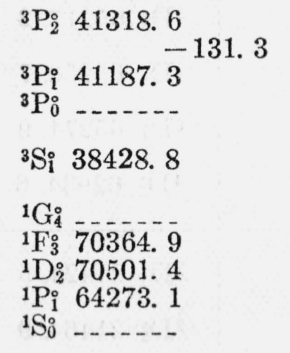 & $\begin{array}{ll}{ }^{1} \mathrm{~F}_{3}^{\circ} & \ldots \\
& \\
{ }^{1} \mathrm{D}_{2}^{\circ} & 51740.9 \\
{ }^{1} \mathrm{P}_{1}^{\circ} & 52650.0\end{array}$ \\
\hline $4 d$ & $\begin{array}{cr}{ }^{5} \mathrm{D}_{4}^{\circ} 37376.2 & 1.2 \\
{ }^{5} \mathrm{D}_{3}^{\circ} 37377.4 & 3.0 \\
{ }^{5} \mathrm{D}_{2}^{\circ} 37380.4 & 1.8 \\
{ }^{5} \mathrm{D}_{\mathrm{i}} 37382.2 & 1.1 \\
{ }^{5} \mathrm{D}_{0}^{\circ} 37383.3 & \\
& \\
{ }^{8} \mathrm{D}_{2}^{\circ} & 30203.5 \\
{ }^{8} \mathrm{D}_{2}^{\circ} 30092.3 & -111.2 \\
{ }^{8} \mathrm{D}_{1} & 30010.2\end{array}$ & 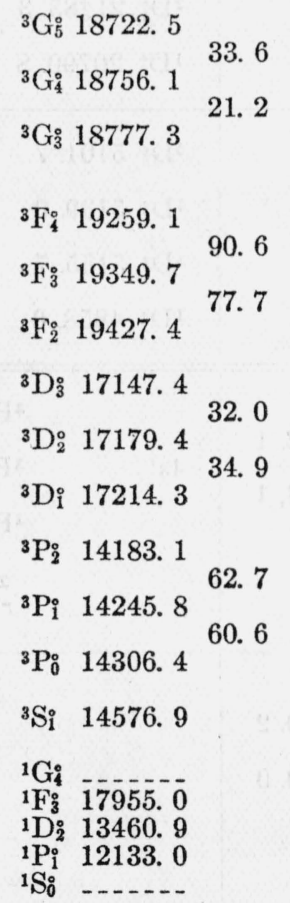 & 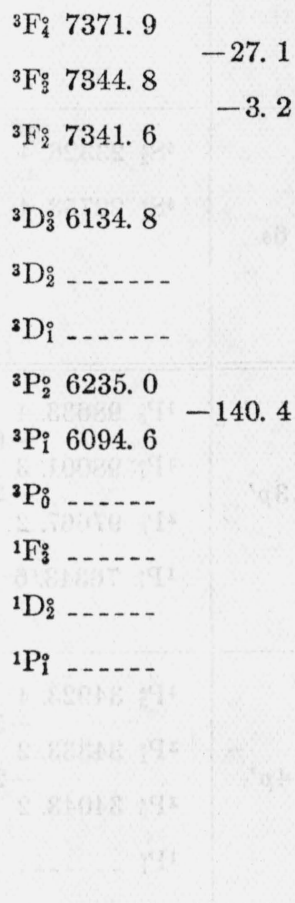 \\
\hline $5 d$ & 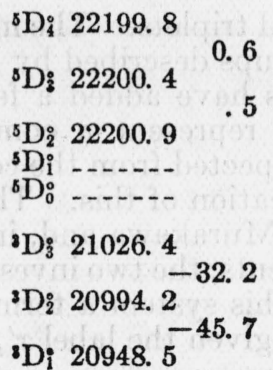 & 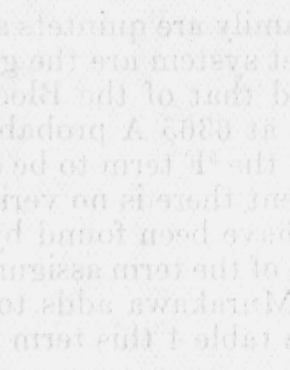 & \\
\hline
\end{tabular}


TABLE 4.-Observed terms of $\mathrm{Cl} I \mathrm{I}-\mathrm{Continued}$

\begin{tabular}{|c|c|c|c|c|}
\hline & Limit: ${ }^{4} \mathrm{~S}=321936$ & Limit $\left\{\begin{array}{l}2 D_{21 / 2}=303816 \\
{ }^{2} D_{1 / 2}=303883\end{array}\right.$ & \multicolumn{2}{|c|}{$\operatorname{Limit}\left\{\begin{array}{l}{ }^{2} \mathrm{P}_{11 / 3}=292029 \\
{ }^{2} \mathrm{P}_{01 / 2}=292124\end{array}\right.$} \\
\hline $4 s$ & 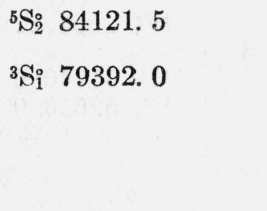 & $\begin{array}{rrr}{ }^{3} \mathrm{D}_{3}^{\circ} & 65217.2 & \\
{ }^{3} \mathrm{D}_{2}^{\circ} & 65256.7 & 39.5 \\
& & \\
& & 18.2 \\
{ }^{3} \mathrm{D}_{1}^{\circ} & 65274.9 & \\
{ }^{1} \mathrm{D}_{2}^{\circ} & 62934.6 & \end{array}$ & $\begin{array}{rr}{ }^{3} \mathrm{P}_{2} & 54122.4 \\
{ }^{3} \mathrm{P}_{1} & 54195.6 \\
{ }^{3} \mathrm{P}_{0} & 54229.9 \\
{ }^{1} \mathrm{P}_{1} & 53377.0\end{array}$ & $\begin{array}{l}73.2 \\
34.3\end{array}$ \\
\hline $5 s$ & $\begin{array}{lll}{ }^{5} \mathrm{~S}_{2} & 39766.9 \\
{ }^{3} \mathrm{~S}_{1} & 38366.9\end{array}$ & $\begin{array}{rrr}{ }^{3} \mathrm{D}_{3}^{\circ} & 21424.5 & \\
{ }^{3} \mathrm{D}_{2}^{\circ} & 21464.9 & \\
& & \\
& & \\
& & \\
{ }^{3} \mathrm{D}_{1}^{\circ} & 21485.3 & \\
{ }^{1} \mathrm{D}_{2} & 20790.8 & \end{array}$ & $\begin{array}{r}{ }^{3} \mathrm{P}_{2}^{\circ} 9551.3 \\
{ }^{3} \mathrm{P}_{1}^{\circ} 9627.7 \\
{ }^{3} \mathrm{P}_{0}^{\circ} 9662.1 \\
{ }^{1} \mathrm{P}_{1} \\
\end{array}$ & $\begin{array}{l}76.4 \\
34.4\end{array}$ \\
\hline $6 s$ & $\begin{array}{ll}{ }^{5} \mathrm{~S}_{2} & 23326.4 \\
{ }^{3} \mathrm{~S}_{1} & 22753.4\end{array}$ & $\begin{array}{rrr}{ }^{3} \mathrm{D}_{3}^{\circ} & 5101.7 & \\
{ }^{3} \mathrm{D}_{2}^{\circ} & 5139.0 & 37.3 \\
{ }^{3} \mathrm{D}_{1}^{\circ} & 5155.7 & 16.7 \\
{ }^{1} \mathrm{D}_{2}^{\circ} & 4858.6 & \end{array}$ & & \\
\hline $3 p^{\prime}$ & 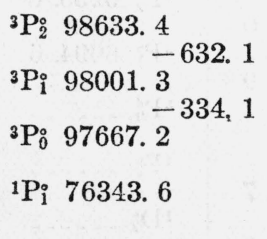 & $\begin{array}{ll}{ }^{8} \mathrm{P}_{2} & 3185 \\
{ }^{\prime} \quad \mathrm{P}_{1} & 3200 \\
{ }^{3} \mathrm{P}_{0} & 3215 \\
x^{\prime} & 277 \\
x^{\prime \prime} & 177\end{array}$ & $\begin{array}{r}143.8 \\
159.3\end{array}$ & \\
\hline $4 p^{\prime}$ & 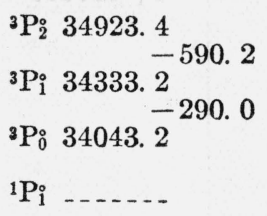 & & & \\
\hline
\end{tabular}

The terms of the ${ }^{4} \mathrm{~S}$ family are quintets and triplets. The important multiplets of the quintet system are the groups described by Paschen. To these, our work and that of the Blochs have added a few more. The faint, diffuse lines at $6365 \mathrm{~A}$ probably represent a combination between $\left({ }^{4} \mathrm{~S}\right) 4 d^{5} \mathrm{D}^{\circ}$ and the ${ }^{5} \mathrm{~F}$ term to be expected from the configuration $\left({ }^{4} \mathrm{~S}\right) 4 f$; but at present there is no verification of this. The triplet terms of the ${ }^{4} \mathrm{~S}$ family have been found by Murakawa and, independently, by us. For most of the term assignments, the two investigations are in agreement, but Murakawa adds to this system a term that he designates as $4 f^{3} \mathrm{~F}$. In table 4 this term is given the label $x^{\prime}$, indicat- 
ing that, for the present, we prefer to leave it unassigned. The reasons for this are stated below. The intercombinations between the two systems of the ${ }^{4} \mathrm{~S}$ family have now been found and Paschen's quintet multiplets, which have stood apart, are here tied up with the extensive triplet systems.

The terms of the ${ }^{2} \mathrm{D}$ family consist of singlets and triplets and constitute the largest group of $\mathrm{Cl}$ II. Some of the strongest lines of the spectrum are accounted for by the combinations of the $3 d$ and $4 \mathrm{~s}$ terms with the $4 p$ terms. Less intense, but equally numerous, groups of lines come from the combinations of the $4 d, 5 s$, and $6 s$ with the $4 p$ terms. Murakawa has reported all the triplet terms in this family from the $4 s, 4 p, 4 d$, and $5 s$ electron configurations, some from the $3 d$ configuration, and a few singlet terms. In addition to these terms, we give in table 4 nearly all the singlets required for these configurations and the rest of the triplets of the $3 d$ group.

The terms of the ${ }^{2} \mathrm{P}$ family are given here for the first time, except $3 d^{3} \mathrm{D}^{\circ}$, which is due to Bowen. The prominent lines of this family, as with the ${ }^{2} \mathrm{D}$ family, arise from the combination of the $4 s$ terms, ${ }^{1} \mathrm{P}^{\circ}$ and ${ }^{3} \mathrm{P}^{\circ}$, with the $4 p$ terms. The $3 d$ terms, although approaching the $4 s$ terms in stability, do not combine as readily with the $4 p$ terms. This is revealed by the nonappearance of the multiplets $3 d{ }^{3} \mathrm{D}^{\circ}-$ $4 p^{3} \mathrm{~F}, 3 d^{3} \mathrm{P}^{\circ}-4 p^{3} \mathrm{D}, 3 d^{3} \mathrm{P}^{\circ}-4 p^{3} \mathrm{~S}$, and only the partial appearance of $3 d^{3} \mathrm{P}^{\circ}-4 p^{3} \mathrm{P}, 3 d^{3} \mathrm{P}^{\circ}-4 s^{\prime}{ }^{3} \mathrm{P}$.

The singlets of both the ${ }^{2} \mathrm{D}$ and ${ }^{2} \mathrm{P}$ families presented some difficulty. After most of the triplet terms described above had been found, the remaining outstanding lines were searched for recurring, constant wave-number differences. Among these lines the differences 7,788.5, $5,651.2$, and $1,774.2 \mathrm{~cm}^{-1}$ were recognized as significant. In particular, two pairs of lines with the difference 7,788 also exhibited the difference $13,409 \mathrm{~cm}^{-1}$ that separated two strong lines in the far ultraviolet. This clue led to the scheme of singlet terms as given in table 4, and further search revealed the intersystem combinations that tied them up with the triplets and quintets.

The terms of the ${ }^{4} \mathrm{~S},{ }^{2} \mathrm{D}$, and ${ }^{2} \mathrm{P}$ families described in the preceding paragraphs result from removal of a $p$ electron from the configuration $3 s^{2} 3 p^{4}$. The configuration $3 s 3 p^{5}$ is also possible and the excitation of $\mathrm{Cl}^{+}$may proceed by removal of the $s$ electron. This yields another family of ${ }^{3} \mathrm{P}^{\circ}$ and ${ }^{1} \mathrm{P}^{\circ}$ terms with $3 p^{5}\left({ }^{2} \mathrm{P}^{\prime}\right)$ of $\mathrm{Cl}$ III as limit. The terms recognized as members of this family are distinguished by the configuration symbols $3 p^{\prime}$ and $4 p^{\prime}$ in table 4 .

After the term assignments discussed above had been made, there remained undesignated three terms, $4 s^{\prime}{ }^{3} \mathrm{P}$ and two single terms $x^{\prime}$ and $x^{\prime \prime}$, for which no origin was obvious. These terms are of even parity and give some rather strong combinations. Murakawa has interpreted the term $x^{\prime}$ as $\left({ }^{4} \mathrm{~S}\right) 4 f^{3} \mathrm{~F}$. If this is correct, then $x^{\prime \prime}$ must be regarded as $\left({ }^{4} \mathrm{~S}\right) 5 f^{3} \mathrm{~F}$, because its behavior is closely similar to that of $x^{\prime}$. In support of this view is the fact that if they result from addition of an $f$ electron to ${ }^{4} \mathrm{~S}$ then they are closely hydrogenic in character, their Rydberg denominators being 3.973 and 4.973 , respectively. However, they do not exhibit the combinatory characteristies of ${ }^{3} \mathrm{~F}$ terms. They combine strongly only with the $3 d^{3} \mathrm{D}$ terms of the ${ }^{4} \mathrm{~S},{ }^{2} \mathrm{D}$, and ${ }^{2} \mathrm{P}$ families. We have photographed the lines in question with high dispersion, in particular the three lines at $2250 \mathrm{~A}$ with the dispersion of $0.5 \mathrm{~A} / \mathrm{mm}$ afforded by the large quartz-prism spectro- 
graph, and find them to be sharp, without shading or satellites. The observational evidence indicates that the terms $x^{\prime}$ and $x^{\prime \prime}$ are single and with inner quantum number 2 . If they belong to the ${ }^{4} \mathrm{~S}$ family, they might be regarded as ${ }^{3} \mathrm{P}_{2}$ terms obtained by addition of $n p$ electrons to ${ }^{4} \mathrm{~S}$, but failure to observe close components of the multiple term argues against this view.

An alternative, though not satisfactory, view is to attribute the origin of the terms $4 s^{\prime}{ }^{3} \mathrm{P}, x^{\prime}$, and $x^{\prime \prime}$ to the configuration $3 s 3 p^{4} 4 s$ resulting from excitation of an $s$ electron out of the ground state of $\mathrm{Cl}^{+}$. Terms of this character are not known in other spectra related to $\mathrm{Cl} \mathrm{II}$, and a weighty objection to this interpretation is the nonappearance in the analysis of the other terms of the configuration, which might reasonably be expected to be present with some prominence.

\section{SERIES AND IONIZATION POTENTIAL}

The ${ }^{5} \mathrm{~S}^{\circ}$ and ${ }^{3} \mathrm{~S}^{\circ}$ terms of the ${ }^{4} \mathrm{~S}$ family form excellent sequences from which to calculate absolute term values. For each series we have observed combinations with terms from the $4 s, 5 s$, and $6 s$ electrons. We may, therefore, rigorously solve the Ritz term formula

$$
m s S=\frac{4 R_{\mathrm{Cl}}}{\{m+\alpha+\beta(m s S)\}^{2}}
$$

and evaluate the constants. For the quintet series, we find $4 s$ ${ }^{5} \mathrm{~S}_{2}=84,150 \mathrm{~cm}^{-1} ; \alpha=-1.6467 ;$ and $\beta=0.8134 \times 10^{-6} ;$ and for the triplet series we find $4 s^{3} \mathrm{~S}_{1}=79,333 \quad \mathrm{~cm}^{-1} ; \quad \alpha=-1.5846$; and $\beta=-0.7917 \times 10^{-6}$.

Similar excellent sequences may be found in the terms of the $\left({ }^{2} \mathrm{D}\right) m s$ groups. As an example, we may cite the series of ${ }^{3} \mathrm{D}_{3}$ terms that converge to ${ }^{2} \mathrm{D}_{21 / 2}$ of $\mathrm{Cl}$ III. From three observed members we solve a Ritz formula similar to the above and find $4 s^{3} \mathrm{D}_{3}=83,411 \mathrm{~cm}^{-1}$; $\alpha=-1.6387$; and $\beta=8 \times 10^{-7}$. This value of $4 s^{3} \mathrm{D}_{3}$, when reckoned from ${ }^{4} \mathrm{~S}$, must be diminished by $18,120 \mathrm{~cm}^{-1}$, the distance between ${ }^{4} \mathrm{~S}$ and ${ }^{2} \mathrm{D}_{21 / 3}$. In the list of ultraviolet lines, table 2 , may be found combinations between the lowest term of $\mathrm{Cl}$ II and the terms $4 s$ ${ }^{5} \mathrm{~S}_{2}^{\circ}, 4 s^{3} \mathrm{~S}_{\mathrm{i}}$, and $4 s^{3} \mathrm{D}_{3}^{\circ}$. These give $192,032,191,938$, and $192,075 \mathrm{~cm}^{-1}$, respectively, for the distance separating the ground states of $\mathrm{Cl}$ II and Cl III. Accordingly, we adopt $192,000 \mathrm{~cm}^{-1}$ for the value of $3 p{ }^{3} \mathrm{P}_{2}$, corresponding to an ionization potential of 23.70 volts. This is in exact agreement with Murakawa's result.

It is well known that series from the $d$ electron are not satisfactorily represented by the Ritz formula. For the ${ }^{5} \mathrm{D}$ terms of $\mathrm{Cl}$ II arising from ${ }^{4} \mathrm{~S}$, we can find only an approximate agreement with the formula. For the ${ }^{3} \mathrm{D}$ terms of the same family, the lack of agreement is quite pronounced, being aggravated by the perturbative influence of the term $3 d^{3} \mathrm{D}$ of the ${ }^{2} \mathrm{D}$ family. The effect of this perturbation is to shift the term $4 d^{3} \mathrm{D}$ from its normal position in the energy scale, and is further manifested in the shifted positions and the anomalous separations of the other terms of the series. This behavior is precisely that described by Shenstone and Russell ${ }^{20}$ for the perturbed series of various elements.

${ }^{20}$ A. G. Shenstone and H. N. Russell, Phys. Rev, 39, 415 (1932). 


\section{COMPARISON WITH SIMILAR SPECTRA}

The spectra $\mathrm{SI}, \mathrm{Cl}$ II, A III, Krv, Cav, etc., form an isoelectronic sequence, which it is instructive to examine in order to check term assignments and to discover the clues leading to new identifications. Partial term analyses have been made for each of these spectra: by Meissner, Bartelt, and Eckstein ${ }^{21}$ and by Ruedy ${ }^{22}$ for SI; by deBruin ${ }^{23}$

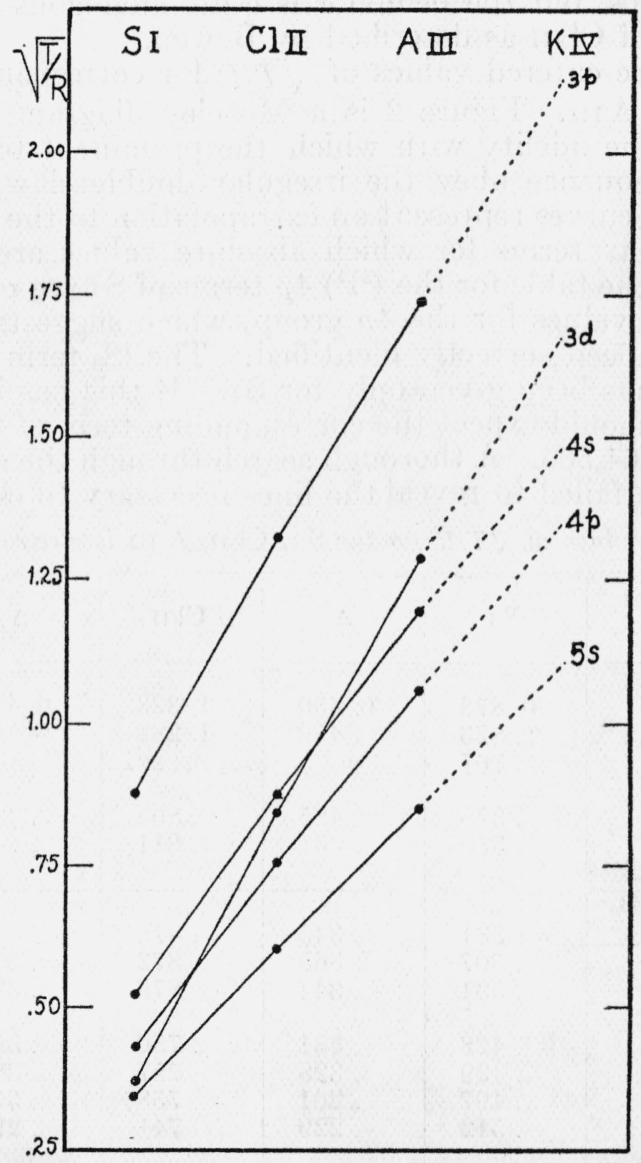

Figure 2.-Moseley diagram for $\mathrm{S}$ I isoelectronic sequence.

for AIII; by Ram ${ }^{24}$, and by Bowen ${ }^{25}$ for KIv and Cav. In only the first three spectra, however, have series been found from which absolute term values may be derived.

A striking feature of the term structure of these spectra is the increasing stability with atomic number of the $3 d$ electron. In $\mathrm{S}_{\mathrm{I}}$ the $3 d$ terms lie in the vicinity of the $4 p$ group and combine with them to give lines, not yet observed, that fall far into the infrared. In Clir, however, as shown in figure 1, the $3 d$ terms approach the $4 s$

\footnotetext{
${ }^{11}$ K. W. Meissner, O. Bartelt, and L. Eckstein, Z. Physik 86, 54 (1933).

22 J. E. Ruedy, Phys. Rev. 44, 757 (1933).

23 T. L. deBruin, Proc. Acad. Sci. Amsterdam 40, 340 (1937).

24 M. Ram, Indian J. Phys. 8, 151 and 163 (1933).

as I. S. Bowen, Phys. Rev. 16, 791 (1934).
} 
group in stability, while in A III and the succeeding spectra the $3 d$ terms have dropped below the $4 s$ groups. An exception to this statement is to be noted for some of the $3 d$ terms of the ${ }^{2} \mathrm{D}$ family. We find that the ${ }^{3} \mathrm{D}^{\circ},{ }^{3} \mathrm{P}^{\circ}$, and ${ }^{3} \mathrm{~S}^{\circ}$ terms of this group, if they have been correctly designated, lie above the $4 p$ terms, and apparently are unrelated to the ${ }^{3} \mathrm{G}^{\circ}$ and ${ }^{3} \mathrm{~F}^{\circ}$ terms. No simple explanation is apparent for this division of the terms of a configuration into two widely separated groups, but the behavior is quite analogous to that of the ( $\left.{ }^{3} \mathrm{P}\right) 3 d$ terms of $\mathrm{Cl}$ III as described by Bowen.

In table 5 are entered values of $\sqrt{T / R}$ for corresponding terms of $\mathrm{S}$, Clir, and Airr. Figure 2 is a Moseley diagram of these data and portrays the fidelity with which the prominent terms of the SI isoelectronic sequence obey the irregular doublet law. The dotted portions of the curves represent an extrapolation to the probable positions of the K Iv terms for which absolute values are still lacking. The entries in the table for the $\left({ }^{2} \mathrm{P}\right) 4 p$ terms of $\mathrm{S}$ I are out of harmony with the other values for the $4 p$ group, which suggests that perhaps they have not been correctly identified. The ${ }^{1} \mathrm{~S}_{0}$ term of the ground configuration has been given only for $\mathrm{S}_{\mathrm{I}}$. If this has been correctly identified, we should expect the corresponding term of $\mathrm{Cl}$ II to have a value close to 164,500 . A thorough search through the available wave length lists has failed to reveal the lines necessary to establish it.

TABLe 5.-Values of $\sqrt{T / R}$ for the $\mathrm{S} \mathrm{I}, \mathrm{Cl}$ II, A III isoelectronic sequence

\begin{tabular}{|c|c|c|c|c|c|}
\hline Term & $\mathrm{S}_{\mathrm{I}}$ & $\Delta$ & $\mathrm{Cl}_{\text {II }}$ & $\Delta$ & A III \\
\hline $\begin{array}{ll}3 p & 3 \mathrm{P} \\
3 p & 1 \mathrm{D} \\
3 p & 1 \mathrm{~S}\end{array}$ & $\begin{array}{r}0.873 \\
.823 \\
.767\end{array}$ & $\begin{array}{r}0.450 \\
.459\end{array}$ & $\begin{array}{l}\text { 1. } 323 \\
\text { 1. } 282 \\
\end{array}$ & 0.413 & 1. 734 \\
\hline $\begin{array}{l}\left({ }^{4} \mathrm{~S}\right) 3 d^{5} \mathrm{D}^{\circ} \\
\left({ }^{4} \mathrm{~S}\right) 3 d^{3} \mathrm{D}^{\circ}\end{array}$ & $\begin{array}{l}.378 \\
.350\end{array}$ & $\begin{array}{l}.485 \\
.461\end{array}$ & $\begin{array}{l}.863 \\
.811\end{array}$ & $\begin{array}{l}.436 \\
.445\end{array}$ & $\begin{array}{l}\text { 1. } 299 \\
\text { 1. } 256\end{array}$ \\
\hline $\begin{array}{l}\left({ }^{4} \mathrm{~S}\right) 4 s^{5} \mathrm{~S}^{\circ} \\
\text { (2D) } 4 s^{3} \mathrm{D}^{\circ} \\
\text { (2) } 4 s^{3} \mathrm{P}^{\circ}\end{array}$ & $\begin{array}{l}.531 \\
.507 \\
.531\end{array}$ & $\begin{array}{l}.345 \\
.365 \\
.344\end{array}$ & $\begin{array}{l}.876 \\
.872 \\
.875\end{array}$ & $\begin{array}{l}.315 \\
.315 \\
.324\end{array}$ & $\begin{array}{l}\text { 1. } 191 \\
\text { 1. } 187 \\
1.199\end{array}$ \\
\hline $\begin{array}{l}\left({ }^{4} \mathrm{~S}\right) 4 p^{5} \mathrm{P} \\
\left({ }^{2} \mathrm{D}\right) 4 p^{3} \mathrm{~F} \\
\left({ }^{2} \mathrm{P}\right) 4 p^{3} \mathrm{D} \\
\left({ }^{2} \mathrm{P}\right) 4 p^{3} \mathrm{P}\end{array}$ & $\begin{array}{l}428 \\
.429 \\
.497 \\
.512\end{array}$ & $\begin{array}{l}331 \\
.328 \\
261 \\
.229\end{array}$ & $\begin{array}{l}.759 \\
.757 \\
.758 \\
.741\end{array}$ & $\begin{array}{l}.309 \\
.308 \\
.308 \\
.297\end{array}$ & $\begin{array}{l}\text { 1. } 068 \\
\text { 1. } 065 \\
1.066 \\
1.038\end{array}$ \\
\hline $\begin{array}{l}\left({ }^{4} \mathrm{~S}\right) 5 s^{5} \mathrm{~S}^{\circ} \\
\left({ }^{2} \mathrm{D}\right) 5 s^{3} \mathrm{D}^{\circ} \\
\left({ }^{2} \mathrm{P}\right) 5 s^{3} \mathrm{P}^{\circ}\end{array}$ & .342 & .260 & $\begin{array}{l}.602 \\
.600 \\
.600\end{array}$ & $\begin{array}{l}.248 \\
.248 \\
.248\end{array}$ & $\begin{array}{l}.850 \\
.848 \\
.848\end{array}$ \\
\hline
\end{tabular}

In conclusion we express our indebtedness to our colleagues at the National Bureau of Standards who have aided us during various phases of the work described in these pages. Particularly do we wish to express our appreciation to I. S. Bowen, J. C. Boyce, and F. Weinberg for making available to us their unpublished observations of chlorine spectra in the Schumann region.

Washington, May 10, 1939. 